Louisiana State University

LSU Digital Commons

$1-15-2013$

\title{
Synthesis and cellular studies of polyamine conjugates of a mercaptomethyl-carboranylporphyrin
}

N. V.S.Dinesh K. Bhupathiraju

Louisiana State University

M. Graça H. Vicente

Louisiana State University

Follow this and additional works at: https://digitalcommons.Isu.edu/chemistry_pubs

\section{Recommended Citation}

Bhupathiraju, N., \& Vicente, M. (2013). Synthesis and cellular studies of polyamine conjugates of a mercaptomethyl-carboranylporphyrin. Bioorganic and Medicinal Chemistry, 21 (2), 485-495.

https://doi.org/10.1016/j.bmc.2012.11.007

This Article is brought to you for free and open access by the Department of Chemistry at LSU Digital Commons. It has been accepted for inclusion in Faculty Publications by an authorized administrator of LSU Digital Commons.

For more information, please contact ir@lsu.edu. 


\title{
Synthesis and Cellular Studies of Polyamine Conjugates of a Mercaptomethyl-carboranylporphyrin'
}

\author{
N.V.S. Dinesh K. Bhupathiraju and M. Graça H. Vicente \\ Louisiana State University, Department of Chemistry, Baton Rouge LA, 70803, USA
}

\begin{abstract}
Seven polyamine conjugates of a tri( $p$-carboranylmethylthio)tetrafluorophenylporphyrin were prepared in high yields by sequential substitution of the $p$-phenyl fluoride of tetrakis(pentafluorophenyl)porphyrin (TPPF), and investigated as boron delivery agents for boron neutron capture therapy (BNCT). The polyamines used were derivatives of the natural-occurring spermine with different lengths of the carbon chains, terminal primary amine groups and, in two of the conjugates, additional aminoethyl moieties. A tri(polyethylene glycol) conjugate was also synthesized for comparison purposes. The polyamine conjugates showed low dark cytotoxicity $\left(\mathrm{IC}_{50}>400 \mu \mathrm{M}\right)$ and low phototoxicity $\left(\mathrm{IC}_{50}>40 \mu \mathrm{M}\right.$ at $\left.1.5 \mathrm{~J} / \mathrm{cm}^{2}\right)$. All polyamine conjugates, with one exception, showed higher uptake into human glioma T98G cells (up to 12-fold) than the PEG conjugate, and localized preferentially in the cell ER, Golgi and the lysosomes. Our results show that spermine derivatives can serve as effective carriers of boronated porphyrins for the BNCT of tumors.
\end{abstract}

\section{Keywords}

BNCT; polyamine; PDT; carborane; porphyrin

\section{Introduction}

Boron-containing porphyrins are promising boron delivery agents for the boron neutron capture therapy (BNCT) of tumors, due to their ability for selective delivery of high amount of boron (> $20 \mu \mathrm{g} / \mathrm{g}$ ) to target tissues with low dark toxicity, and their long retention times in tumors. ${ }^{1,2}$ BNCT is a binary cancer treatment that involves the irradiation of ${ }^{10} \mathrm{~B}$-containing tumor cells with low energy thermal or epithermal neutrons to produce high linear energy transfer (high-LET) alpha and lithium-7 particles, and releasing 2.4 MeV of kinetic energy, according to the equation below: ${ }^{3-5}$

\footnotetext{
${ }^{1}$ Abbreviations: PDT, photodynamic therapy; BNCT, boron neutron capture therapy; TPPF, 5,10,15,20-tetrakis(2,3,4,5,6pentafluorophenyl)porphyrin; DMSO, dimethylsulfoxide; THF, tetrahydrofuran; TFA, trifluoroacetic acid; LET, linear energy transfer; PBS, phosphate buffered saline; FBS, fetal bovine serum; ER, endoplasmic reticulum.

(C) 2012 Elsevier Ltd. All rights reserved.

*To whom correspondence should be addressed. Phone: 225-578-7405. Fax: 225-578-3458. vicente@1su.edu. Supplementary data Available: Details are provided on the syntheses of the polyamines and mercaptomethyl- $p$-carborane, HPLC traces and conditions, cellular images for PEG-conjugate 9, and fluorescence emission spectra of conjugates 2-9.

Publisher's Disclaimer: This is a PDF file of an unedited manuscript that has been accepted for publication. As a service to our customers we are providing this early version of the manuscript. The manuscript will undergo copyediting, typesetting, and review of the resulting proof before it is published in its final citable form. Please note that during the production process errors may be discovered which could affect the content, and all legal disclaimers that apply to the journal pertain.
} 


$$
{ }^{10} \mathrm{~B}+{ }^{1} \mathrm{n} \rightarrow{ }^{7} \mathrm{Li}^{3+}+{ }^{4} \mathrm{He}^{2+}+\gamma+2.4 \mathrm{MeV}
$$

The ${ }^{10} \mathrm{~B}$ nucleus has a much higher neutron capture cross section (3838 barns, 1 barn = $\left.10^{-24} \mathrm{~cm}^{2}\right)$, than ${ }^{12} \mathrm{C}(0.0034$ barn $),{ }^{1} \mathrm{H}(0.33$ barn $)$ and ${ }^{14} \mathrm{~N}$ (1.8 barns), which make up approximately $96 \%$ of tissues, thus minimizing damage to normal cells. Indeed, BNCT has the potential to be highly selective, able to destroy ${ }^{10} \mathrm{~B}$-containing malignant cells in the presence of normal boron-free cells, due to the limited path range $(5-9 \mu \mathrm{m})$ of the high-LET cytotoxic particles in tissues. The only two boron compounds currently in BNCT clinical trials in Japan, Finland and Sweden are BSH (sodium mercaptoundecahydro-closododecaborate) and BPA ( $L$-4-dihydroxy-borylphenylalanine), used either alone or in combination; although improved patient survival has been reported using these drugs, ${ }^{6-9}$ high doses of these agents, particularly BPA, are typically required to achieve a therapeutic response, and both BSH and BPA have only low selectivity for tumor cells and low retention times in tumors. On the other hand, boronated porphyrins have shown increase tumor selectivity and retention times compared with BSH and BPA. Furthermore, boroncontaining porphyrins retain the fluorescence and photosensitizing properties characteristic of this type of macrocycle, allowing the use of photodynamic therapy (PDT) as adjuvant treatment for BNCT, and facilitating the detection of tissue-localized boron and treatment planning. ${ }^{2,10}$ PDT involves the activation of a tumor-localized photosensitizer with red light, producing reactive oxygen species, such as ${ }^{1} \mathrm{O}_{2}$, that cause irreversible photodamage to malignant tissues. ${ }^{11,12}$ Two porphyrin derivatives, Photofrin and Visudyne, are FDAapproved for the PDT treatment of cancer of the lung, digestive tract, genitourinary tract, melanoma, Barrett's esophagus and, in the latter case, the wet form of age-related macular degeneration. ${ }^{13,14}$ On the other hand, BNCT has been investigated for tumors that are difficult to treat by PDT, such as glioblastoma multiforme, due in part to limited light penetration and/or insufficient tissue oxygenation. Due to the large amount of ${ }^{10} \mathrm{~B}$ in tumor (20-35 $\mu \mathrm{g} / \mathrm{g}$, depending on the boron microdistribution) required for effective BNCT, recent research has centered on the synthesis of boron-containing porphyrins of high boron content, containing stable boron clusters and a tumor-targeting moiety, such as a peptide. ${ }^{15,16}$ Among the boron clusters, the negatively charged closo- $\mathrm{B}_{12} \mathrm{H}_{12}{ }^{2-}$ anion, the carborane derivatives closo- $\mathrm{CB}_{11} \mathrm{H}_{12}{ }^{-}$and nido- $\mathrm{C}_{2} \mathrm{~B}_{9} \mathrm{H}_{11}{ }^{-}$, and metallo-bis(dicarbollides) such as $\left[3,3^{\prime}-\mathrm{Co}\left(1,2-\mathrm{C}_{2} \mathrm{~B}_{9} \mathrm{H}_{11}\right)_{2}\right]^{-}$, have been the most common clusters used for attachment to porphyrins because of their high boron content, known chemistry, amphiphilic properties, and their high photochemical, kinetic and hydrolytic stabilities. As a result, most of the boron-containing porphyrin derivatives reported to date are negatively charged, ${ }^{2}$ although positively charged boronated agents potentially target the most sensitive tumor cell organelles, e.g. mitochondria and nuclei, and important biomolecules such as DNA and RNA. ${ }^{17}$ Furthermore, the amount of ${ }^{10} \mathrm{~B}$ required for effective BNCT can be substantially reduced if it localizes near or within the cell nucleus. ${ }^{18}$ Herein we report the synthesis and investigation of a series of amphiphilic closo-carboranylmethylthioporphyrins containing fluoride and conjugated to various polyamines, which are protonated under physiologic conditions. Fluorinated porphyrins have been previously shown to have increased photodynamic activities compared with their non-fluorinated analogs, ${ }^{19,20}$ and could facilitate imaging (using ${ }^{19} \mathrm{~F}-\mathrm{NMR}$ and/or ${ }^{18} \mathrm{~F}$-PET in addition to porphyrin fluorescence) and treatment outcome. ${ }^{21}$ On the other hand, polyamines are known to be essential for cell growth and differentiation, and are found in high concentrations in rapidly proliferating tumor cells, due to an up-regulated polyamine transport system. ${ }^{22-24}$ As a consequence, polyamine conjugation to porphyrins ${ }^{1}$ and other drugs ${ }^{29-34}$ is an attractive strategy for increasing tumor selectivity, uptake and overall biological efficacy. Several boroncontaining polyamines have been synthesized and shown to bind to DNA $;{ }^{35-41}$ although significant toxicity was observed for some of these polycationic molecules, new low 
cytotoxicity derivatives were synthesized but no additional biological investigations are yet reported. Therefore, conjugation of polyamines to a boron-containing porphyrin could potentially increase boron uptake into tumor cells, favor DNA binding and overall BNCT efficacy. In this paper seven polyamines were selected for conjugation to a pre-formed carboranylporphyrin, containing between 3 and 5 amine groups and 2, 3 or 4 carbon spacers between the nitrogens. The uptake and toxicity of the polyamine-porphyrin conjugates were evaluated in human glioma T98G cells and compared with those of a tripegylated analog.

\section{Experimental Section}

\section{Chemistry}

Reactions that are sensitive were conducted under argon atmosphere in oven-dried glassware. Commercially available reagents and solvents (HPLC grade) were purchased from Sigma-Aldrich, Acros Organics and used without further purification. p-carborane was purchased from Katchem, Inc. Anhydrous methanol was prepared by distillation from magnesium turnings and was stored under nitrogen over $3 \mathrm{~A}^{0}$ molecular sieves. Anhydrous THF was prepared by distillation from sodium and benzophenone. Analytical thin-layerchromatography (TLC) was performed on polyester backed TLC plates 254 (pre-coated, 200 $\mu \mathrm{m}$, Sorbent Technologies). Silica gel 60 (70-230 mesh, Merk) used for column chromatography and silica gel TLC plates $(0.2 \mathrm{~mm}$ thickness) were purchased from Sorbent Technologies. ${ }^{1} \mathrm{H}$ NMR and ${ }^{13} \mathrm{C}$ NMR spectra were obtained using a Bruker AV-4 $400 \mathrm{MHz}$ spectrometer; chemical shifts are expressed in $\mathrm{ppm} .{ }^{19} \mathrm{~F}$ NMR spectra were obtained using a Bruker DPX-250 $250 \mathrm{MHz}$ spectrometer; chemical shifts are expressed in ppm. Electronic absorption spectra were measured on a Perkin Elmer Lambda 35 UV-vis spectrophotometer. Mass analysis was conducted at the LSU Mass Spectrometry Facility on a Bruker Omniflex MALDI-TOF mass spectrometer and exact masses were obtained from HRMS-ESI on an Applied Biosystems QSTAR XL. Melting points were measured on a Thomas hoover melting point apparatus. Reversed-phase HPLC was performed on a Waters system including a 2545 quaternary gradient module pump with a $2489 \mathrm{UV}$-vis detector and a fraction collector III. An analytical column (4.6X250 mm-XBridgeTM BED300 C18 $5 \mu \mathrm{m}$ ) was used for the purification of all polyamine conjugates (2-8) and a stepwise gradient from 0 to $100 \%$ Buffer B in the first $10 \mathrm{~min}$ to $50 \% \mathrm{~B}$ and $50 \% \mathrm{C}$ in next $10 \mathrm{~min}$ to $100 \% \mathrm{~B}$ in next 10 min. A stepwise gradient 10-90\% Buffer B with Buffer A was used for the PEG conjugate 9. Buffer A ( $0.1 \%$ TFA, H2O), Buffer B ( $0.1 \%$ TFA, acetonitrile), Buffer C $(0.1 \%$ TFA, acetone). All Boc-protected polyamines were synthesized as previously described. ${ }^{42}$ 1-Mercaptomethyl- $p$-carborane was prepared from $p$-carborane, as described in literature. ${ }^{45}$

\section{5,10,15-Tri(p-carboranylmethylthiotetrafluorophenyl)-20-} pentafluorophenylporphyrin (1)-To a solution of TPPF (19.52 $\mathrm{mg}, 0.02 \mathrm{mmol})$ in 2 $\mathrm{ml}$ of dry DMF was added anhydrous $\mathrm{K}_{2} \mathrm{CO}_{3}(16.6 \mathrm{mg}, 0.12 \mathrm{mmol})$, mercaptomethyl- $p$ carborane $(15.2 \mathrm{mg}, 0.08 \mathrm{mmol})$. The reaction mixture was stirred at room temperature for $48 \mathrm{~h}$. The resulting solution was diluted with ethyl acetate $(50 \mathrm{~mL})$ and washed with brine (2 x $50 \mathrm{~mL}$ ). The organic layer was dried over anhydrous sodium sulfate, the solvents evaporated under reduced pressure and the resulting residue was dissolved in dichloromethane $(10 \mathrm{~mL})$. A saturated solution of $\mathrm{Zn}(\mathrm{OAc})_{2}$ in methanol $(10 \mathrm{~mL})$ was added and the resulting mixture stirred at room temperature for $24 \mathrm{~h}$. The solvents were evaporated under reduced pressure and the resulting residue was purified by prep-TLC using chloroform/petroleum ether 1:1 to give $23.3 \mathrm{mg}(30 \%)$ of desired zinc(II) porphyrin, $\mathrm{mp}>$ $300^{0} \mathrm{C}$. UV-Vis (DMSO) $\lambda_{\max }\left(\varepsilon / \mathrm{M}^{-1} \mathrm{~cm}^{-1}\right) 426$ (444 400), 555 (43 800). ${ }^{1} \mathrm{H}-\mathrm{NMR}$ $\left(\mathrm{CDCl}_{3}, 400 \mathrm{MHz}\right): \delta 8.93(\mathrm{~s}, 8 \mathrm{H}, \beta-\mathrm{H}), 3.45$ (s, 6H, $\left.-\mathrm{SCH}_{2}\right), 2.19-3.33(\mathrm{~m}, 33 \mathrm{H}, \mathrm{BH}, \mathrm{CH})$. MS (MALDI-TOF) $\mathrm{m} / \mathrm{z} 1550.789$ [M], calcd for $\mathrm{ZnC}_{53} \mathrm{H}_{47} \mathrm{~F}_{17} \mathrm{~N}_{4} \mathrm{~B}_{30} \mathrm{~S}_{3}=1550.494$. The $\mathrm{Zn}$ (II) porphyrin ( $23.3 \mathrm{mg}, 0.015 \mathrm{mmol}$ ) was dissolved in $2 \mathrm{~mL}$ of TFA/chloroform 1:1 and 
stirred at room temperature overnight. After removal of the solvents under vacuum, the title porphyrin was obtained in quantitative yield. $\mathrm{mp}>300^{0} \mathrm{C}$. UV-vis (DMSO) $\lambda_{\max }\left(\varepsilon / \mathrm{M}^{-1}\right.$ $\mathrm{cm}^{-1}$ ) 416 (468 900), 511 (45 900), 555 (18 700), 585 (11 200), 650 (8 300). ${ }^{1} \mathrm{H}-\mathrm{NMR}$ $\left(\mathrm{CDCl}_{3}, 400 \mathrm{MHz}\right): \delta 9.02(\mathrm{~s}, 2 \mathrm{H}, \beta-\mathrm{H}), 8.90(\mathrm{~s}, 6 \mathrm{H}, \beta-\mathrm{H}), 3.46\left(\mathrm{~s}, 6 \mathrm{H},-\mathrm{SCH}_{2}\right), 1.78-2.96$ (m, 33H, BH \& CH), -2.87 (s, 2H, NH). ${ }^{13} \mathrm{C}-\mathrm{NMR}\left(\mathrm{CDCl}_{3}, 100 \mathrm{MHz}\right): \delta 147.82,145.34$, 143.62, 141.07, 138.89, 136.35, 120.85, 115.54, 104.64, 81.88, 59.23, 14.13. ${ }^{19} \mathrm{~F}-\mathrm{NMR}$ (acetone- $\left.\mathrm{d}_{6}, 233.3 \mathrm{MHz}\right): \delta-135.7(\mathrm{~m}, 6 \mathrm{~F}),-139.8(\mathrm{~m}, 6 \mathrm{~F}),-140.4(\mathrm{~d}, J=15.7 \mathrm{~Hz}, 2 \mathrm{~F})$, -156.2 (t, $J=19.0 \mathrm{~Hz}, 1 \mathrm{~F}$ ), -165.0 (d, J = 16.1 Hz, 2F). MS (MALDI-TOF) $\mathrm{m} / \mathrm{z} 1485.672$ [M], calcd for $\mathrm{C}_{53} \mathrm{H}_{49} \mathrm{~F}_{17} \mathrm{~N}_{4} \mathrm{~B}_{30} \mathrm{~S}_{3}=1485.565$.

Conjugate 2-To porphyrin $1(14.9 \mathrm{mg}, 0.01 \mathrm{mmol})$ were added (N1,N3,N6-tri-tertbutoxycarbonyl)-1,8-di-amino-3,6-diazaoctane ${ }^{42}(6.7 \mathrm{mg}, 0.015 \mathrm{mmol})$ and NMP (2 $\mathrm{mL})^{43,44}$ and the mixture was heated at $100{ }^{\circ} \mathrm{C}$ for $4 \mathrm{~h}$. After cooling to room temperature, the solution was diluted with ethyl acetate $(50 \mathrm{~mL})$ and washed with brine $(5 \times 50 \mathrm{~mL})$. The organic layer was dried over anhydrous sodium sulfate, the solvents removed under vacuum and the resulting reddish brown residue purified by silica gel column chromatography using dichloromethane for elution, followed by dichloromethane/ethyl acetate 9:1. The Bocprotected conjugate was obtained $(18.6 \mathrm{mg})$ in $96 \%$ yield, $\mathrm{mp}=292-294{ }^{\circ} \mathrm{C}$. UV-Vis (DMSO) $\lambda_{\max }\left(\varepsilon / \mathrm{M}^{-1} \mathrm{~cm}^{-1}\right) 416$ (401 700), 510 (28 400), 555 (22 000), 585 (11 000), 650 (10 500). ${ }^{1} \mathrm{H}-\mathrm{NMR}\left(\mathrm{CDCl}_{3}, 400 \mathrm{MHz}\right): \delta 9.06(\mathrm{~s}, 2 \mathrm{H}, \beta-\mathrm{H}), 8.91(\mathrm{~s}, 6 \mathrm{H}, \beta-\mathrm{H}), 3.33-3.53$ $\left(\mathrm{m}, 16 \mathrm{H}, \mathrm{SCH}_{2}, \mathrm{NCH}_{2}\right), 2.72-2.79\left(\mathrm{~m}, 2 \mathrm{H}, \mathrm{CH}_{2}\right), 1.89-2.71(\mathrm{~m}, 33 \mathrm{H}, \mathrm{BH}$ and $\mathrm{CH}), 1.58(\mathrm{~s}$, $\left.9 \mathrm{H},{ }^{\mathrm{t}} \mathrm{Bu}\right), 1.52\left(\mathrm{~s}, 9 \mathrm{H},{ }^{\mathrm{t}} \mathrm{Bu}\right), 1.46\left(\mathrm{~s}, 9 \mathrm{H},{ }^{\mathrm{t}} \mathrm{Bu}\right),-2.86(\mathrm{~s}, 2 \mathrm{H}, \mathrm{NH}) .{ }^{13} \mathrm{C}-\mathrm{NMR}\left(\mathrm{CDCl}_{3}, 100\right.$ MHz): $\delta 155.92,155.61,147.54,146.84,143.23,142.87,137.89,136.15,119.85,115.44$, $103.84,81.58,79.78,79.67,78.54,59.4352 .67,49.96,47.19,46.35,45.43,40.34,38.98$, 30.78, 28.14, 14.23. ${ }^{19} \mathrm{~F}-\mathrm{NMR}$ (acetone- $\left.\mathrm{d}_{6}, 233.3 \mathrm{MHz}\right): \delta-135.8(\mathrm{~m}, 6 \mathrm{~F}),-139.9(\mathrm{~m}, 6 \mathrm{~F})$, $-144.2(\mathrm{~d}, J=14.8 \mathrm{~Hz}, 2 \mathrm{~F}),-162.3(\mathrm{~d}, J=15.6 \mathrm{~Hz}, 2 \mathrm{~F})$. The Boc-protected conjugate was dissolved in TFA/dichloromethane 1:1 (2 mL) and stirred at room temperature for $6 \mathrm{~h}$. After removal of the solvent under vacuum, the resulting residue was purified by HPLC to give conjugate $2(14.7 \mathrm{mg})$ in $95 \%$ yield; $\mathrm{mp}>300^{\circ} \mathrm{C}$. HPLC $t_{\mathrm{R}}=27.01 \mathrm{~min}$. UV-vis (DMSO) $\lambda_{\max }\left(\varepsilon / \mathrm{M}^{-1} \mathrm{~cm}^{-1}\right) 416$ (591 400), 510 (52 600), 555 (21 900), 585 (11 400), 650 (10 000). ${ }^{1} \mathrm{H}-\mathrm{NMR}\left(\mathrm{CDCl}_{3}, 400 \mathrm{MHz}\right)$ : $\delta 8.75-9.75$ (br s, $\left.8 \mathrm{H}, \beta-\mathrm{H}\right), 3.33-3.69\left(\mathrm{~m}, 16 \mathrm{H}, \mathrm{SCH}_{2}\right.$ $\left.\& \mathrm{CH}_{2}\right), 2.35-3.11\left(\mathrm{~m}, 35 \mathrm{H}, \mathrm{CH}_{2}, \mathrm{BH}, \mathrm{CH}\right) .{ }^{19} \mathrm{~F}-\mathrm{NMR}$ (acetone- $\left.\mathrm{d}_{6}, 233.3 \mathrm{MHz}\right): \delta-135.6$ $(\mathrm{m}, 6 \mathrm{~F}),-139.8(\mathrm{~m}, 6 \mathrm{~F}),-144.2(\mathrm{~d}, J=15.4 \mathrm{~Hz}, 2 \mathrm{~F}),-162.4(\mathrm{~d}, J=15.9 \mathrm{~Hz}, 2 \mathrm{~F})$. HRMS (ESI-TOF) $\mathrm{m} / \mathrm{z}$ found $1612.7414\left[\mathrm{M}+\mathrm{H}^{+}\right], 806.3720\left[\mathrm{M}+2 \mathrm{H}^{+}\right]^{2+}$, calcd for $\mathrm{C}_{59} \mathrm{H}_{64} \mathrm{~F}_{16} \mathrm{~N}_{8} \mathrm{~B}_{30} \mathrm{~S}_{3}=1612.7008,\left[\mathrm{C}_{59} \mathrm{H}_{64} \mathrm{~F}_{16} \mathrm{~N}_{8} \mathrm{~B}_{30} \mathrm{~S}_{3}\right]^{2+}=806.3504$.

Conjugate 3-A similar procedure was used to that described above for conjugate 2, using porphyrin 1 (14.9 mg, $0.01 \mathrm{mmol})$, (N1,N3,N6,N9-tetra-tert-butoxycarbonyl)-1,11-diamino-3,6,9-triazaundecane ${ }^{42}(8.85 \mathrm{mg}, 0.015 \mathrm{mmol})$ and NMP $(2 \mathrm{~mL}) .{ }^{43,44}$ The Bocprotected conjugate was obtained $(20 \mathrm{mg})$ in $96 \%$ yield, $\mathrm{mp}=292-295{ }^{\circ} \mathrm{C}$. UV-vis (DMSO) $\lambda_{\max }\left(\varepsilon / \mathrm{M}^{-1} \mathrm{~cm}^{-1}\right) 416$ (592 500), 510 (21 200), 555 (18 000), 585 (9 400), 650 (8 600); ${ }^{1} \mathrm{H}-\mathrm{NMR}\left(\mathrm{CDCl}_{3}, 400 \mathrm{MHz}\right): \delta 9.05$ (s, $\left.2 \mathrm{H}, \beta-\mathrm{H}\right), 8.90(\mathrm{~s}, 6 \mathrm{H}, \beta-\mathrm{H}), 3.25-3.45(\mathrm{~m}$, $\left.20 \mathrm{H}, \mathrm{SCH}_{2}, \mathrm{NCH}_{2}\right), 1.75-2.98\left(\mathrm{~m}, 33 \mathrm{H}, \mathrm{CH}_{2}, \mathrm{BH}, \mathrm{CH}\right), 1.57$ (s, 9H, $\left.{ }^{\mathrm{t}} \mathrm{Bu}\right), 1.50$ (s, $\left.18 \mathrm{H},{ }^{\mathrm{t}} \mathrm{Bu}\right), 1.43\left(\mathrm{~s}, 9 \mathrm{H},{ }^{\mathrm{t}} \mathrm{Bu}\right),-2.86(\mathrm{~s}, 2 \mathrm{H}, \mathrm{NH}) .{ }^{13} \mathrm{C}-\mathrm{NMR}\left(\mathrm{CDCl}_{3}, 100 \mathrm{MHz}\right): \delta 156.92$, 156.16, 155.39, 147.64, 145.60, 143.30, 141.16, 138.06, 135.68, 120.86, 115.30, 103.94, $81.89,80.69,79.14,77.24,60.41,59.22,50.21,47.45,47.36,45.42,40.62,39.47,29.71$, 28.43, 28.21, 24.83, 21.06, 14.21. ${ }^{19} \mathrm{~F}-\mathrm{NMR}$ (acetone- $\left.\mathrm{d}_{6}, 233.3 \mathrm{MHz}\right): \delta-135.9(\mathrm{~m}, 6 \mathrm{~F})$, $-139.8(\mathrm{~m}, 6 \mathrm{~F}),-144.3(\mathrm{~d}, \mathrm{~J}=15.3 \mathrm{~Hz}, 2 \mathrm{~F}),-162.5(\mathrm{~d}, \mathrm{~J}=16.0 \mathrm{~Hz}, 2 \mathrm{~F})$. The Boc protected conjugate was deprotected using TFA in dichloromethane, as described above, and conjugate 3 was obtained $(15.1 \mathrm{mg})$ in $95 \%$ yield after HPLC purification; $\mathrm{mp}>300{ }^{\circ} \mathrm{C}$; HPLC $t_{R}=26.68 \mathrm{~min}$. UV-vis (DMSO) $\lambda_{\max }\left(\varepsilon / \mathrm{M}^{-1} \mathrm{~cm}^{-1}\right) 416$ (596 900), 510 (54 500), 555 (24 400), 585 (12 500), 650 (10 900). ${ }^{1} \mathrm{H}-\mathrm{NMR}\left(\mathrm{CDCl}_{3}, 400 \mathrm{MHz}\right): \delta 8.88-9.60$ (br s, 
8H, $\beta-\mathrm{H}), 3.33-3.72\left(\mathrm{~m}, 20 \mathrm{H}, \mathrm{SCH}_{2}, \mathrm{CH}_{2}\right), 2.22-3.35$ (m, 35H, $\left.\mathrm{CH}_{2}, \mathrm{BH}, \mathrm{CH}\right) .{ }^{19} \mathrm{~F}-\mathrm{NMR}$ (acetone- $\left.\mathrm{d}_{6}, 233.3 \mathrm{MHz}\right): \delta-135.9(\mathrm{~m}, 6 \mathrm{~F}),-139.9(\mathrm{~m}, 6 \mathrm{~F}),-144.4(\mathrm{~d}, J=15.1 \mathrm{~Hz}, 2 \mathrm{~F})$, $-162.3(\mathrm{~d}, J=16.4 \mathrm{~Hz}, 2 \mathrm{~F})$. HRMS (ESI-TOF) $\mathrm{m} / \mathrm{z}$ found $1656.8000\left[\mathrm{M}+\mathrm{H}^{+}\right], 828.4016$ [M $+2 \mathrm{H}]^{2+}$, calcd for $\left[\mathrm{C}_{61} \mathrm{H}_{68} \mathrm{~F}_{16} \mathrm{~N}_{9} \mathrm{~B}_{30} \mathrm{~S}_{3}\right]=1656.7418,\left[\mathrm{C}_{61} \mathrm{H}_{68} \mathrm{~F}_{16} \mathrm{~N}_{9} \mathrm{~B}_{30} \mathrm{~S}_{3}\right]^{2+}=828.3709$.

Conjugate 4-A similar procedure was used to that described above for conjugate $\mathbf{2}$, using porphyrin 1 (14.9 mg, $0.01 \mathrm{mmol})$, (N1,N3,N6,N9,N12-penta-tert-butoxycarbonyl)-1,14-diamino-3,6,9,12-tetraazatetradecane ${ }^{42}(11.0 \mathrm{mg}, 0.015 \mathrm{mmol})$ and NMP $(2 \mathrm{~mL}){ }^{43,44}$ The Boc-protected conjugate was obtained $(21.4 \mathrm{mg})$ in $96 \%$ yield, $\mathrm{mp}=293-296^{\circ} \mathrm{C}$; UV-vis (DMSO) $\lambda_{\max }\left(\varepsilon / \mathrm{M}^{-1} \mathrm{~cm}^{-1}\right) 416$ (456 000), 510 (24 000), 555 (21 000), 585 (12 500), 650 (10 300). ${ }^{1} \mathrm{H}-\mathrm{NMR}\left(\mathrm{CDCl}_{3}, 400 \mathrm{MHz}\right): \delta 9.06$ (s, $\left.2 \mathrm{H}, \beta-\mathrm{H}\right), 8.91$ (s, $\left.6 \mathrm{H}, \beta-\mathrm{H}\right), 3.22-3.56$ (m, 24H, $\left.\mathrm{SCH}_{2}, \mathrm{NCH}_{2}\right), 2.72-2.79$ (m, 2H, $\left.\mathrm{CH}_{2}\right), 1.8-3.15$ (m, 35H, $\left.\mathrm{CH}_{2}, \mathrm{BH}, \mathrm{CH}\right), 1.58$ (s, $\left.9 \mathrm{H},{ }^{\mathrm{t}} \mathrm{Bu}\right), 1.48-1.51\left(\mathrm{~m}, 27 \mathrm{H},{ }^{\mathrm{t}} \mathrm{Bu}\right), 1.43\left(\mathrm{~s}, 9 \mathrm{H},{ }^{\mathrm{t}} \mathrm{Bu}\right),-2.86(\mathrm{~s}, 2 \mathrm{H}, \mathrm{NH}) .{ }^{13} \mathrm{C}-\mathrm{NMR}\left(\mathrm{CDCl}_{3}\right.$, $100 \mathrm{MHz}$ ): $\delta$ 156.99, 156.14, 155.33, 147.64, 145.49, 145.16, 143.53, 138.09, 137.90, $121.06,120.88,115.31,103.94,81.90,80.95,80.63,80.23,80.04,79.12,60.41,59.23$, 47.39, 45.43, 39.45, 38.76, 37.65, 28.91, 21.06, 14.21. ${ }^{19} \mathrm{~F}-\mathrm{NMR}$ (acetone- $\mathrm{d}_{6}, 233.3 \mathrm{MHz}$ ): $\delta-135.8(\mathrm{~m}, 6 \mathrm{~F}),-139.6(\mathrm{~m}, 6 \mathrm{~F}),-144.0(\mathrm{~d}, J=15.4 \mathrm{~Hz}, 2 \mathrm{~F}),-162.2(\mathrm{~d}, J=16.1 \mathrm{~Hz}, 2 \mathrm{~F})$. The Boc protected conjugate was deprotected using TFA in dichloromethane, as described above, and conjugate 4 was obtained $(15.5 \mathrm{mg})$ in $95 \%$ yield after HPLC purification, $\mathrm{mp}>$ $300^{0} \mathrm{C}$; HPLC $\mathrm{t}_{\mathrm{R}}=26.14 \mathrm{~min}$. UV-vis (DMSO) $\lambda_{\max }\left(\varepsilon / \mathrm{M}^{-1} \mathrm{~cm}^{-1}\right) 416$ (552 300), 510 (45 900), 555 (18 400), 585 (14 100), 650 (5 700). ${ }^{1} \mathrm{H}-\mathrm{NMR}\left(\mathrm{CDCl}_{3}, 400 \mathrm{MHz}\right): \delta 8.78-9.61$ (br s, 8H, $\beta-\mathrm{H}$ ), $3.25-3.87$ (m, 24H, $\mathrm{SCH}_{2}, \mathrm{CH}_{2}$ ), 2.09-3.18 (m, 35H, $\left.\mathrm{CH}_{2}, \mathrm{BH}, \mathrm{CH}\right) .{ }^{19} \mathrm{~F}-$ NMR (acetone- $\left.\mathrm{d}_{6}, 233.3 \mathrm{MHz}\right): \delta-135.4(\mathrm{~m}, 6 \mathrm{~F}),-139.7(\mathrm{~m}, 6 \mathrm{~F}),-144.3(\mathrm{~d}, J=15.8 \mathrm{~Hz}$, $2 \mathrm{~F}),-162.5(\mathrm{~d}, J=16.3 \mathrm{~Hz}, 2 \mathrm{~F})$. HRMS (ESI-TOF) $\mathrm{m} / z$ found $1698.8939\left[\mathrm{M}+\mathrm{H}^{+}\right]$, $849.9461\left[\mathrm{M}+2 \mathrm{H}^{+}\right]^{2+}, 566.9658\left[\mathrm{M}+3 \mathrm{H}^{+}\right]^{3+}$; calcd for $\mathrm{C}_{63} \mathrm{H}_{72} \mathrm{~F}_{16} \mathrm{~N}_{10} \mathrm{~B}_{30} \mathrm{~S}_{3}=1698.7639$, $\left[\mathrm{C}_{63} \mathrm{H}_{72} \mathrm{~F}_{16} \mathrm{~N}_{10} \mathrm{~B}_{30} \mathrm{~S}_{3}\right]^{2+}=849.3819,\left[\mathrm{C}_{63} \mathrm{H}_{72} \mathrm{~F}_{16} \mathrm{~N}_{10} \mathrm{~B}_{30} \mathrm{~S}_{3}\right]^{3+}=566.2546$.

Conjugate 5-A similar procedure was used to that described above for conjugate $\mathbf{2}$, using porphyrin 1 (14.9 mg, $0.01 \mathrm{mmol}$ ), (N1,N3,N7-tri-tert-butoxycarbonyl)-1,9-di-amino-3,7diazanonane $^{42}(6.7 \mathrm{mg}, 0.015 \mathrm{mmol})$ and NMP $(2 \mathrm{~mL}) .{ }^{43,44}$ The Boc-protected conjugate was obtained (18.7 mg) in $96 \%$ yield, $\mathrm{mp}=280-283^{\circ} \mathrm{C}$. UV-vis (DMSO) $\lambda_{\max }\left(\varepsilon / \mathrm{M}^{-1}\right.$ $\mathrm{cm}^{-1}$ ) 416 (512 300), 510 (35 900), 555 (16 700), 585 (12 100), 650 (9 000). ${ }^{1} \mathrm{H}-\mathrm{NMR}$ $\left(\mathrm{CDCl}_{3}, 400 \mathrm{MHz}\right): \delta 9.06(\mathrm{~s}, 2 \mathrm{H}, \beta-\mathrm{H}), 8.91(\mathrm{~s}, 6 \mathrm{H}, \beta-\mathrm{H}), 3.47\left(\mathrm{~s}, 6 \mathrm{H}, \mathrm{SCH}_{2}\right), 3.3-3.45(\mathrm{~m}$, $\left.10 \mathrm{H}, \mathrm{NCH}_{2}\right), 1.73-3.09\left(\mathrm{~m}, 37 \mathrm{H}, \mathrm{CH}_{2}, \mathrm{BH}, \mathrm{CH}\right), 1.58\left(\mathrm{~s}, 9 \mathrm{H},{ }^{\mathrm{t}} \mathrm{Bu}\right), 1.51\left(\mathrm{~s}, 9 \mathrm{H},{ }^{\mathrm{t}} \mathrm{Bu}\right), 1.45$ $\left(\mathrm{s}, 9 \mathrm{H},{ }^{\mathrm{t}} \mathrm{Bu}\right),-2.86(\mathrm{~s}, 2 \mathrm{H}, \mathrm{NH}) .{ }^{13} \mathrm{C}-\mathrm{NMR}\left(\mathrm{CDCl}_{3}, 100 \mathrm{MHz}\right): \delta 156.07,147.63,145.30$, $143.01,135.67,130.73,120.86,115.29,103.94,81.88,80.63,80.12,79.28,60.41,59.22$, 47.08, 45.59, 40.62, 39.62, 28.06, 14.21. ${ }^{19}$ F-NMR (acetone- $\left.\mathrm{d}_{6}, 233.3 \mathrm{MHz}\right): \delta-135.9(\mathrm{~m}$, $6 \mathrm{~F}),-139.4(\mathrm{~m}, 6 \mathrm{~F}),-144.6(\mathrm{~d}, J=15.2 \mathrm{~Hz}, 2 \mathrm{~F}),-162.6(\mathrm{~d}, J=15.8 \mathrm{~Hz}, 2 \mathrm{~F})$. The Boc protected conjugate was deprotected using TFA in dichloromethane, as described above, and conjugate 5 was obtained $(14.8 \mathrm{mg}$ ) in 95\% yield after HPLC purification; $\mathrm{mp}=292-294$ ${ }^{\circ} \mathrm{C}$. HPLC $t_{\mathrm{R}}=26.80 \mathrm{~min}$; UV-vis (DMSO) $\lambda_{\max }\left(\varepsilon / \mathrm{M}^{-1} \mathrm{~cm}^{-1}\right) 416$ (482 600), 510 (41 800), 555 (17 696), 585 (10 863), 650 (6 763). ${ }^{1} \mathrm{H}-\mathrm{NMR}\left(\mathrm{CDCl}_{3}, 400 \mathrm{MHz}\right): \delta 8.90-9.70$ (br s, 8H, $\beta-\mathrm{H}), 3.60$ (s, 6H, SCH$), 3.33-3.53\left(\mathrm{~m}, 10 \mathrm{H}, \mathrm{CH}_{2}\right), 2.24-3.11\left(\mathrm{~m}, 37 \mathrm{H}, \mathrm{CH}_{2}\right.$, $\mathrm{BH}, \mathrm{CH}) .{ }^{19} \mathrm{~F}-\mathrm{NMR}$ (acetone- $\left.\mathrm{d}_{6}, 233.3 \mathrm{MHz}\right): \delta-135.4(\mathrm{~m}, 6 \mathrm{~F}),-139.6(\mathrm{~m}, 6 \mathrm{~F}),-144.8(\mathrm{~d}$, $J=15.4 \mathrm{~Hz}, 2 \mathrm{~F}),-162.1(\mathrm{~d}, J=15.9 \mathrm{~Hz}, 2 \mathrm{~F})$. HRMS (ESI-TOF) $\mathrm{m} / \mathrm{z}$ found 1626.7602 [M $\left.+\mathrm{H}^{+}\right], 813.8820\left[\mathrm{M}+2 \mathrm{H}^{+}\right]^{2+}$, calcd for $\mathrm{C}_{60} \mathrm{H}_{66} \mathrm{~F}_{16} \mathrm{~N}_{8} \mathrm{~B}_{30} \mathrm{~S}_{3}=1626.7262$, $\left[\mathrm{C}_{60} \mathrm{H}_{66} \mathrm{~F}_{16} \mathrm{~N}_{8} \mathrm{~B}_{30} \mathrm{~S}_{3}\right]^{2+}=813.3631$.

Conjugate 6-A similar procedure was used to that described above for conjugate $\mathbf{2}$, using porphyrin 1 (14.9 mg, $0.01 \mathrm{mmol})$, (N1,N4,N8-tri-tert-butoxycarbonyl)-1,11-di-amino-4,8diazaundecane ${ }^{42}(7.34 \mathrm{mg}, 0.015 \mathrm{mmol})$ and NMP $(2 \mathrm{~mL}) .{ }^{43,44}$ The Boc-protected conjugate was obtained $\left(19.0 \mathrm{mg}\right.$ ) in $96 \%$ yield, $\mathrm{mp}=270-274^{\circ} \mathrm{C}$; UV-vis (DMSO) $\lambda_{\max }$ 
$\left(\varepsilon / \mathrm{M}^{-1} \mathrm{~cm}^{-1}\right.$ ) 416 (496 100), 510 (39 600), 555 (18 600), 585 (10 800), $650(9300) .{ }^{1} \mathrm{H}-$ NMR $\left(\mathrm{CDCl}_{3}, 400 \mathrm{MHz}\right)$ : $\delta 9.04(\mathrm{~s}, 2 \mathrm{H}, \beta-\mathrm{H}), 8.91$ (s, $\left.6 \mathrm{H}, \beta-\mathrm{H}\right), 3.47$ (s, $\left.6 \mathrm{H}, \mathrm{SCH}_{2}\right), 3.14-$ $3.33\left(\mathrm{~m}, 10 \mathrm{H}, \mathrm{NCH}_{2}\right), 1.71-3.05\left(\mathrm{~m}, 41 \mathrm{H}, \mathrm{CH}_{2}, \mathrm{BH}, \mathrm{CH}\right), 1.56\left(\mathrm{~s}, 9 \mathrm{H},{ }^{\mathrm{t}} \mathrm{Bu}\right), 1.51(\mathrm{~s}$, $\left.9 \mathrm{H},{ }^{\mathrm{t}} \mathrm{Bu}\right), 1.45\left(\mathrm{~s}, 9 \mathrm{H},{ }^{\mathrm{t}} \mathrm{Bu}\right),-2.85(\mathrm{~s}, 2 \mathrm{H}, \mathrm{NH}) .{ }^{13} \mathrm{C}-\mathrm{NMR}\left(\mathrm{CDCl}_{3}, 100 \mathrm{MHz}\right): \delta 156.05$, 147.63, 145.15, 142.43, 141.09, 135.65, 130.95, 121.05, 115.30, 103.94, 81.89, 80.05, $79.88,60.41,59.22,44.99,40.62,38.78,36.54,31.89,31.76,30.78,21.06,14.21 .{ }^{19}$ F-NMR (acetone- $\left.\mathrm{d}_{6}, 233.3 \mathrm{MHz}\right): \delta-135.3(\mathrm{~m}, 6 \mathrm{~F}),-139.7(\mathrm{~m}, 6 \mathrm{~F}),-144.5(\mathrm{~d}, J=15.4 \mathrm{~Hz}, 2 \mathrm{~F})$, -162.3 (d, $J=15.9 \mathrm{~Hz}, 2 \mathrm{~F})$. The Boc protected conjugate was deprotected using TFA in dichloromethane, as described above, and conjugate 6 was obtained $(15.0 \mathrm{mg})$ in $95 \%$ yield after HPLC purification, $\mathrm{mp}=284-287^{\circ} \mathrm{C}$. $\mathrm{HPLC} \mathrm{t}_{\mathrm{R}}=26.35 \mathrm{~min}$. UV-vis (DMSO) $\lambda_{\max }$ $\left(\varepsilon / \mathrm{M}^{-1} \mathrm{~cm}^{-1}\right) 417$ (453 300), 510 (37 500), 555 (14 600), 585 (11 000), $650(9700) .{ }^{1} \mathrm{H}-$ NMR (CDCl $3,400 \mathrm{MHz}$ ): $\delta 8.81-9.82$ (br s, $8 \mathrm{H}, \beta-\mathrm{H}), 3.33-3.83\left(\mathrm{~m}, 16 \mathrm{H}, \mathrm{SCH}_{2}, \mathrm{NCH}_{2}\right.$ ), 2.06-3.29 (m, 41H, $\left.\mathrm{CH}_{2}, \mathrm{BH}, \mathrm{CH}\right) .{ }^{19} \mathrm{~F}-\mathrm{NMR}$ (acetone- $\left.\mathrm{d}_{6}, 233.3 \mathrm{MHz}\right): \delta-135.6(\mathrm{~m}, 6 \mathrm{~F})$, $-139.4(\mathrm{~m}, 6 \mathrm{~F}),-144.4$ (d, $J=15.6 \mathrm{~Hz}, 2 \mathrm{~F}),-162.3$ (d, $J=16.0 \mathrm{~Hz}, 2 \mathrm{~F})$. HRMS (ESI-TOF) $\mathrm{m} / \mathrm{z}$ found $1654.7848\left[\mathrm{M}+\mathrm{H}^{+}\right], 827.8940\left[\mathrm{M}+2 \mathrm{H}^{+}\right]^{2+}$, calcd for $\left[\mathrm{C}_{62} \mathrm{H}_{70} \mathrm{~F}_{16} \mathrm{~N}_{8} \mathrm{~B}_{30} \mathrm{~S}_{3}\right]=$ 1654.7577, $\left[\mathrm{C}_{62} \mathrm{H}_{70} \mathrm{~F}_{16} \mathrm{~N}_{8} \mathrm{~B}_{30} \mathrm{~S}_{3}\right]^{2+}=827.3788$.

Conjugate 7-A similar procedure was used to that described above for conjugate $\mathbf{2}$, using porphyrin 1 (14.9 mg, $0.01 \mathrm{mmol})$, (N1,N4,N9-tri-tert-butoxycarbonyl)-1,12-di-amino-4,9diazadodecane ${ }^{42}(7.5 \mathrm{mg}, 0.015 \mathrm{mmol})$ and NMP $(2 \mathrm{~mL}){ }^{43,44}$ The Boc-protected conjugate was obtained $(19.1 \mathrm{mg})$ in $96 \%$ yield, $\mathrm{mp}=276-278{ }^{\circ} \mathrm{C}$. UV-vis (DMSO) $\lambda_{\max }\left(\varepsilon / \mathrm{M}^{-1}\right.$ $\mathrm{cm}^{-1}$ ) 416 (490 800), 510 (39 600), 555 (18 600), 585 (11 900), 650 (9 900). ${ }^{1} \mathrm{H}-\mathrm{NMR}$ $\left(\mathrm{CDCl}_{3}, 400 \mathrm{MHz}\right): \delta 9.04$ (s, $\left.2 \mathrm{H}, \beta-\mathrm{H}\right), 8.91$ (s, $\left.6 \mathrm{H}, \beta-\mathrm{H}\right), 3.47$ (s, $\left.6 \mathrm{H}, \mathrm{SCH}_{2}\right), 3.33-3.43$ (m, $10 \mathrm{H}, \mathrm{CH}_{2}$ ), 1.57-3.13 (m, 43H, $\left.\mathrm{CH}_{2}, \mathrm{BH}, \mathrm{CH}\right), 1.55$ (s, $\left.9 \mathrm{H},{ }^{\mathrm{t}} \mathrm{Bu}\right), 1.50$ (s, $\left.9 \mathrm{H},{ }^{\mathrm{t}} \mathrm{Bu}\right), 1.45$ (s, 9H, $\left.{ }^{\mathrm{t}} \mathrm{Bu}\right),-2.86$ (s, 2H, NH). ${ }^{13} \mathrm{C}-\mathrm{NMR}\left(\mathrm{CDCl}_{3}, 100 \mathrm{MHz}\right): \delta 156.10,147.77,145.64$, 143.30, 139.15, 137.01, 121.05, 115.30, 103.94, 81.89, 79.90, 77.28, 59.22, 49.42, 46.82, 40.62, 30.68, 29.72, 29.58, 17.66. ${ }^{19} \mathrm{~F}-\mathrm{NMR}$ (acetone- $\left.\mathrm{d}_{6}, 233.3 \mathrm{MHz}\right): \delta-135.4(\mathrm{~m}, 6 \mathrm{~F})$, -139.4 (m, 6F), -144.5 (d, $J=15.3 \mathrm{~Hz}, 2 \mathrm{~F}),-162.1$ (d, $J=16.2 \mathrm{~Hz}, 2 \mathrm{~F})$. The Boc protected conjugate was deprotected using TFA in dichloromethane, as described above, and conjugate 7 was obtained $(15.2 \mathrm{mg})$ in 95\% yield after HPLC purification; $\mathrm{mp}=284-287$ ${ }^{\circ} \mathrm{C}$. HPLC $\mathrm{t}_{\mathrm{R}}=26.17 \mathrm{~min}$. UV-vis (DMSO) $\lambda_{\max }\left(\varepsilon / \mathrm{M}^{-1} \mathrm{~cm}^{-1}\right) 416$ (491 875), 510 (40 656), 555 (15 991), 585 (10 700), 650 (6 500). ${ }^{1} \mathrm{H}-\mathrm{NMR}\left(\mathrm{CDCl}_{3}, 400 \mathrm{MHz}\right): \delta 8.90-9.24$ (br s, $8 \mathrm{H}, \beta-\mathrm{H}$ ), 3.61 (s, $\left.6 \mathrm{H}, \mathrm{SCH}_{2}\right), 1.89-3.47$ (m, 53H, $\left.\mathrm{NCH}_{2}, \mathrm{CH}_{2}, \mathrm{BH}, \mathrm{CH}\right) .{ }^{19} \mathrm{~F}-\mathrm{NMR}$ (acetone- $\left.\mathrm{d}_{6}, 233.3 \mathrm{MHz}\right): \delta-135.5(\mathrm{~m}, 6 \mathrm{~F}),-139.8(\mathrm{~m}, 6 \mathrm{~F}),-144.4(\mathrm{~d}, J=15.4 \mathrm{~Hz}, 2 \mathrm{~F})$, $-162.2(\mathrm{~d}, J=15.9 \mathrm{~Hz}, 2 \mathrm{~F})$. HRMS (ESI-TOF) $\mathrm{m} / \mathrm{z}$ found $1668.8031\left[\mathrm{M}+\mathrm{H}^{+}\right], 834.9022[\mathrm{M}$ $\left.+2 \mathrm{H}^{+}\right]^{2+}$, calcd for $\left[\mathrm{C}_{63} \mathrm{H}_{72} \mathrm{~F}_{16} \mathrm{~N}_{8} \mathrm{~B}_{30} \mathrm{~S}_{3}\right]=1668.7734,\left[\mathrm{C}_{63} \mathrm{H}_{72} \mathrm{~F}_{16} \mathrm{~N}_{8} \mathrm{~B}_{30} \mathrm{~S}_{3}\right]^{2+}=834.3867$.

Conjugate 8-A similar procedure was used to that described above for conjugate $\mathbf{2}$, using porphyrin 1 (14.9 mg, $0.01 \mathrm{mmol}$ ), (N1,N4,N7-tri-tert-butoxycarbonyl)-1,10-di-amino-4,7diazaoctane $^{42}(7.1 \mathrm{mg}, 0.015 \mathrm{mmol})$ and NMP $(2 \mathrm{~mL}) .{ }^{43,44}$ The Boc-protected conjugate was obtained (18.8 mg) in $96 \%$ yield, $\mathrm{mp}=289-292{ }^{\circ} \mathrm{C}$. UV-vis (DMSO) $\lambda_{\max }\left(\varepsilon / \mathrm{M}^{-1}\right.$ $\mathrm{cm}^{-1}$ ) 416 (480 800), 510 (41 500), 555 (18 900), 585 (11 600), 650 (8 900). ${ }^{1} \mathrm{H}-\mathrm{NMR}$ $\left(\mathrm{CDCl}_{3}, 400 \mathrm{MHz}\right): \delta 9.03$ (s, $\left.2 \mathrm{H}, \beta-\mathrm{H}\right), 8.90(\mathrm{~s}, 6 \mathrm{H}, \beta-\mathrm{H}), 3.33-3.68\left(\mathrm{~m}, 14 \mathrm{H}, \mathrm{SCH}_{2}\right.$, $\left.\mathrm{NCH}_{2}\right), 1.6-3.1\left(\mathrm{~m}, 41 \mathrm{H}, \mathrm{CH}_{2}, \mathrm{BH}, \mathrm{CH}\right), 1.56\left(\mathrm{~s}, 9 \mathrm{H},{ }^{\mathrm{t}} \mathrm{Bu}\right), 1.52\left(\mathrm{~s}, 9 \mathrm{H},{ }^{\mathrm{t}} \mathrm{Bu}\right), 1.45(\mathrm{~s}$, $\left.9 \mathrm{H},{ }^{\mathrm{t}} \mathrm{Bu}\right),-2.86(\mathrm{~s}, 2 \mathrm{H}, \mathrm{NH}) .{ }^{13} \mathrm{C}-\mathrm{NMR}\left(\mathrm{CDCl}_{3}, 100 \mathrm{MHz}\right): \delta 156.10,155.89,147.63$, $145.15,143.65,140.89,137.78,121.04,115.29,103.94,81.87,80.19,79.87,77.89,60.40$, $59.22,45.76,45.34,44.95,40.62,36.78,36.54,31.56,28.96,28.65,28.50,21.05$, 14.20. ${ }^{19}$ F-NMR (acetone- $\left.\mathrm{d}_{6}, 233.3 \mathrm{MHz}\right): \delta-135.4(\mathrm{~m}, 6 \mathrm{~F}),-139.8(\mathrm{~m}, 6 \mathrm{~F}),-144.2(\mathrm{~d}, J=$ $15.5 \mathrm{~Hz}, 2 \mathrm{~F}),-162.3(\mathrm{~d}, J=16.1 \mathrm{~Hz}, 2 \mathrm{~F})$. The Boc protected conjugate was deprotected using TFA in dichloromethane, as described above, and conjugate $\mathbf{8}$ was obtained (15.0 mg) in $95 \%$ yield after HPLC purification; $\mathrm{mp}=295-298^{\circ} \mathrm{C}$. HPLC $t_{\mathrm{R}}=26.77 \mathrm{~min}$. UV-vis (DMSO) $\lambda_{\max }\left(\varepsilon / \mathrm{M}^{-1} \mathrm{~cm}^{-1}\right) 417$ (438 225), 510 (40 593), 555 (19 538), 585 (12 560), 650 
(9 800). ${ }^{1} \mathrm{H}-\mathrm{NMR}\left(\mathrm{CDCl}_{3}, 400 \mathrm{MHz}\right): \delta 8.78-9.24$ (br s, $\left.8 \mathrm{H}, \beta-\mathrm{H}\right), 3.60\left(\mathrm{~s}, 6 \mathrm{H}, \mathrm{SCH}_{2}\right)$, 3.33-3.53 (m, 8H, $\mathrm{NCH}_{2}$ ), 2.13-3.29 (m, 41H, $\left.\mathrm{CH}_{2}, \mathrm{BH}, \mathrm{CH}\right) .{ }^{19} \mathrm{~F}-\mathrm{NMR}$ (acetone- $\mathrm{d}_{6}, 233.3$ MHz): $\delta-135.6(\mathrm{~m}, 6 \mathrm{~F}),-139.6(\mathrm{~m}, 6 \mathrm{~F}),-144.4(\mathrm{~d}, J=15.6 \mathrm{~Hz}, 2 \mathrm{~F}),-162.4(\mathrm{~d}, J=16.1$ $\mathrm{Hz}, 2 \mathrm{~F}$ ). HRMS (ESI-TOF) $\mathrm{m} / \mathrm{z}$ found $1640.7763\left[\mathrm{M}+\mathrm{H}^{+}\right], 820.8882\left[\mathrm{M}+2 \mathrm{H}^{+}\right]^{2+}$, calcd for $\left[\mathrm{C}_{61} \mathrm{H}_{67} \mathrm{~F}_{16} \mathrm{~N}_{8} \mathrm{~B}_{30} \mathrm{~S}_{3}\right]=1640.7323,\left[\mathrm{C}_{61} \mathrm{H}_{67} \mathrm{~F}_{16} \mathrm{~N}_{8} \mathrm{~B}_{30} \mathrm{~S}_{3}\right]=820.3661$.

Conjugate 9-A similar procedure was used to that described above for conjugate $\mathbf{2}$, using porphyrin 1 (14.9 mg, $0.01 \mathrm{mmol}$ ), tert-butyl-12-amino-4,7,10-trioxadodecanoate ( $4.1 \mathrm{mg}$, $0.015 \mathrm{mmol})$ and NMP $(2 \mathrm{~mL}) .{ }^{43,44}$ The Boc-protected conjugate was obtained $(16.8 \mathrm{mg})$ in $95 \%$ yield, $\mathrm{mp}=289-292{ }^{\circ} \mathrm{C}$; UV-vis (DMSO) $\lambda_{\max }\left(\varepsilon / \mathrm{M}^{-1} \mathrm{~cm}^{-1}\right) 416(460800), 510$ (38 450), 555 (16 700), 585 (10 200), 650 (8 100). ${ }^{1} \mathrm{H}-\mathrm{NMR}\left(\mathrm{CDCl}_{3}, 400 \mathrm{MHz}\right): \delta 9.02$ (s, $2 \mathrm{H}, \beta-\mathrm{H}), 8.90(\mathrm{~s}, 6 \mathrm{H}, \beta-\mathrm{H}), 3.89\left(\mathrm{~s}, 4 \mathrm{H}, \mathrm{CH}_{2}\right), 3.68-3.82\left(\mathrm{~m}, 12 \mathrm{H}, \mathrm{CH}_{2}\right), 1.67-3.10(\mathrm{~m}$, $33 \mathrm{H}, \mathrm{BH}, \mathrm{CH}), 1.43$ (s, 9H, $\left.{ }^{\mathrm{t}} \mathrm{Bu}\right),-2.88(\mathrm{~s}, 2 \mathrm{H}, \mathrm{NH}) .{ }^{13} \mathrm{C}-\mathrm{NMR}\left(\mathrm{CDCl}_{3}, 100 \mathrm{MHz}\right): \delta$ $171.23,149.78,149.51,145.42,145.28,143.77,131.17,125.50,120.45,120.07,119.23$, $118.86,114.49,101.13,100.60,80.16,79.93,53.45,46.56,35.85,27.41 .{ }^{19} \mathrm{~F}-\mathrm{NMR}$ (acetone- $\left.\mathrm{d}_{6}, 233.3 \mathrm{MHz}\right): \delta-135.4(\mathrm{~m}, 6 \mathrm{~F}),-139.6(\mathrm{~m}, 6 \mathrm{~F}),-144.8(\mathrm{~d}, J=15.5 \mathrm{~Hz}, 2 \mathrm{~F})$, $-162.3(\mathrm{~d}, J=16.1 \mathrm{~Hz}, 2 \mathrm{~F})$. HRMS (MALDI-TOF) $\mathrm{m} / \mathrm{z}$ found $1747.822[\mathrm{M}]^{+}$, calcd for $\mathrm{C}_{66} \mathrm{H}_{75} \mathrm{~F}_{16} \mathrm{~N}_{5} \mathrm{~B}_{30} \mathrm{~S}_{3} \mathrm{O}_{5}[\mathrm{M}]^{+}=1747.746$. The Boc protected conjugate was deprotected using TFA in dichloromethane, as described above, and conjugate $\mathbf{9}$ was obtained (15.4 mg) in $95 \%$ yield after $\mathrm{HPLC}$ purification; $\mathrm{mp}=295-298^{\circ} \mathrm{C}$; $\mathrm{HPLC} \mathrm{t}_{\mathrm{R}}=51.21 \mathrm{~min}$. UV-vis (DMSO) $\lambda_{\max }\left(\varepsilon / \mathrm{M}^{-1} \mathrm{~cm}^{-1}\right) 417$ (430 200), 510 (36 700), 555 (18 500), 585 (10 500), 650 (8 800). ${ }^{1} \mathrm{H}-\mathrm{NMR}\left(\mathrm{CDCl}_{3}, 400 \mathrm{MHz}\right): \delta 8.88-9.32(\mathrm{br} \mathrm{s}, 8 \mathrm{H}, \beta-\mathrm{H}), 3.92\left(\mathrm{~s}, 4 \mathrm{H}, \mathrm{CH}_{2}\right), 3.68$ $3.82\left(\mathrm{~m}, 18 \mathrm{H}, \mathrm{CH}_{2}, \mathrm{SCH}_{2}\right), 2.01-3.31(\mathrm{~m}, 33 \mathrm{H}, \mathrm{BH}, \mathrm{CH}) .{ }^{19} \mathrm{~F}-\mathrm{NMR}$ (acetone- $\mathrm{d}_{6}, 233.3$ MHz): $\delta-135.5$ (m, 6F), -139.8 (m, 6F), -144.6 (d, $J=15.6 \mathrm{~Hz}, 2 \mathrm{~F}),-162.4$ (d, $J=16.2$ $\mathrm{Hz}, 2 \mathrm{~F}$ ). HRMS (MALDI-TOF) $\mathrm{m} / z$ found $1691.758[\mathrm{M}]^{+}$, calcd for $\mathrm{C}_{62} \mathrm{H}_{67} \mathrm{~F}_{16} \mathrm{~N}_{5} \mathrm{~B}_{30} \mathrm{~S}_{3} \mathrm{O}_{5}$ $[\mathrm{M}]^{+}=1691.684$.

\section{Octanol-water partition coefficients}

The partition coefficients $(\log P)$ were measured at room temperature by adding $0.3 \mathrm{~mL}$ of a porphyrin stock solution in DMSO $(0.333 \mathrm{mM})$ to a $4 \mathrm{~mL}$ volumetric tube containing 2.0 $\mathrm{mL}$ of HEPES buffer $(50 \mu \mathrm{M}, \mathrm{pH} 7.4)$, followed by addition of $2.0 \mathrm{~mL}$ of 1-octanol. ${ }^{46}$ After vortexing for $5 \mathrm{~min}$, the phases were separated by centrifugation. An aliquot of $0.3 \mathrm{~mL}$ from each layer was diluted with $2 \mathrm{~mL}$ of methanol and the absorbance was read on a Perkin Elmer Lambda $35 \mathrm{UV}-\mathrm{V}$ is spectrophotometer with $10 \mathrm{~mm}$ path length quartz cuvettes.

\section{Cell Studies}

All tissue culture medium and reagents were purchased from Invitrogen (Carlsbad, CA). Human glioma T98G cells were purchased from ATCC and cultured in ATCC-formulated Eagle's Minimum Essential Medium containing 10\% FBS and 1\% antibiotic (Penicillin Streptomycin). The cells were split twice weekly to maintain a sub-confluent stock. All compound solutions were filter-sterilized using a $0.22 \mu \mathrm{m}$ syringe filter.

3.1. Dark Cytotoxicity-10,000 T98G cells were plated per well in a Costar 96 well plate and allowed to grow $36 \mathrm{~h}$. Porphyrin stock solutions $(32 \mathrm{mM})$ were prepared in DMSO and then diluted into final working concentrations $(25,50,100,200,400 \mu \mathrm{M})$. The cells were exposed to increasing concentrations of porphyrin up to $400 \mu \mathrm{M}$ and incubated overnight. The loading medium was removed and the cells washed with $100 \mu \mathrm{L}$ PBS. Then medium containing Cell Titer Blue (Promega) $120 \mu \mathrm{L}$ was added as per manufacturer's instructions. After incubating for $4 \mathrm{~h}$ the cytotoxicity was then measured by reading the fluorescence at $520 / 584 \mathrm{~nm}$ using a BMG FLUOstar plate reader. The signal was normalized to $100 \%$ viable (untreated) cells and $0 \%$ viable (treated with $0.2 \%$ saponin from Sigma) cells. 
3.2. Phototoxicity-The T98G cells were prepared as described above for the dark cytotoxicity assay and treated with porphyrin concentrations of $0,6.25,12.5,25,50$, and 100 $\mu \mathrm{M}$. After compound loading, the medium was removed and replaced with medium containing $50 \mathrm{mM}$ HEPES pH 7.4. The cells were exposed to a NewPort light system with $175 \mathrm{~W}$ halogen lamp for $20 \mathrm{~min}$, filtered through a water filter to provide approximated 1.5 $\mathrm{J} / \mathrm{cm}^{2}$ light dose. The cells were kept cool by placing the culture on a 50C Echotherm chilling/heating plate (Torrey Pines Scientific, Inc.). The cells were returned to the incubator overnight and assayed for viability as described above for the dark cytotoxicity experiment and adding medium containing Cell Titer Blue to determine the toxicity of the compounds.

3.3. Time-Dependent Cellular Uptake-The $\mathrm{T} 98 \mathrm{G}$ cells were prepared as described above for the dark cytotoxicity assay. The cells were exposed to $10 \mu \mathrm{M}$ of each conjugate for $0,1,2,4,8$, and $24 \mathrm{~h}$. At the end of the incubation time the loading medium was removed and the cells were washed with $200 \mu \mathrm{L}$ PBS. The cells were solubilized upon addition of $100 \mu \mathrm{L}$ of $0.25 \%$ Triton X-100 (Calbiochem) in PBS. To determine the porphyrin concentration, fluorescence emission was read at 415/650 nm (excitation/ emission) using a BMG FLUOstar plate reader. The cell numbers were quantified using the CyQuant cell proliferation assay (Invitrogen) as per the manufacturer's instructions, and the uptake was expressed in terms of $\mathrm{nM}$ compound per cell.

3.4. Microscopy-The HEp2 cells were incubated in a glass bottom 6-well plate (MatTek) and allowed to grow for $48 \mathrm{~h}$. The cells were then exposed to $10 \mu \mathrm{M}$ of each porphyrin conjugate for $6 \mathrm{~h}$. Organelle tracers were obtained from In- vitrogen and used at the following concentrations: LysoSensor Green $50 \mathrm{~nm}$, MitoTracker Green $250 \mathrm{~nm}$, ER Tracker Blue/white $100 \mathrm{~nm}$, and BODIPY FL C5 Ceramide $1 \mathrm{~mm}$. The organelle tracers were diluted in medium and the cells were incubated concurrently with porphyrin conjugate and tracers for 30 min before washing 3 times with PBS and microscopy. Images were acquired using a Leica DM RXA2 upright microscope with 40 X NA 0.8dip objective lens and DAPI, GFP and Texas Red filter cubes (Chroma Technologies).

\section{Results and Discussion}

\section{Synthesis and characterization}

TPPF is a commercially available porphyrin that can easily be synthesized in multi-gram scale using a published procedure, ${ }^{47}$ and functionalized via nucleophilic substitution of the $p$-fluoro phenyl groups. ${ }^{48-50}$ The boron cluster used for attachment to TPPF was the 1,12dicarba-closo-dodecaborane, also designated $p$-carborane, rather than the most common 1,2dicarba-closo-dodecaborane or $o$-carborane analog, because of the lower reactivity and higher stability of $p$-carboranes toward base deboronation and degradation. ${ }^{10,51}$ The reaction of TPPF with 1-mercaptomethyl- $p$-carborane ${ }^{45}$ in the presence of $\mathrm{K}_{2} \mathrm{CO}_{3}$ in DMF at room temperature gave a mixture of $p$-carboranyl-containing porphyrins that were very difficult to separate. The highest yields of the tri-substituted porphyrin 1 were obtained when 4 equiv. of 1-mercaptomethyl- $p$-carborane were used relative to TPPF. To facilitate purification, $\mathrm{Zn}$ (II) was inserted by reaction with $\mathrm{Zn}$ (II) acetate in methanol, and after purification by preparative TLC the zinc metal was quantitatively removed using TFA in chloroform. Tri(mercaptomethyl- $p$-carboranyltetrafluorophenyl)pentafluorophenylporphyrin $\mathbf{1}$ was obtained in $30 \%$ overall yield.

The $p$-fluoro phenyl group of porphyrin 1 underwent nucleophilic substitution with the primary amino group of Boc-protected polyamines ${ }^{42}$ and commercially available tertbutyl-12-amino-4,7,10-trioxadodecanoate, ${ }^{43,44}$ as shown in Scheme 1. Deprotection of the Boc and tert-butyl protecting groups using TFA in dichloromethane, gave conjugates 2-9 in 
$91 \%$ overall yields, after reversed-phase HPLC purification. This methodology affords the targeted polyamine-porphyrin conjugates in higher yields than those normally obtained via solid-phase coupling and other methodologies used to conjugate polyamines to porphyrins. $^{25-27}$ The polyamines chosen for conjugation to porphyrin 1 contain between 2 and 4 secondary amine groups and terminal primary amine groups, with various lengths of the intermediate carbon chains. Conjugate $\mathbf{7}$ contains a spermine group, which is a naturallyoccurring polyamine with a 3-4-3 carbon backbone; all other polyamines are derivatives of spermine with different lengths of the carbon chains, of the 2-2-2 (2), 2-3-2 (5), 3-2-3 (8) or 3-3-3 (6) types, and conjugates 3 and 4 contain one or two additional aminoethyl moieties, respectively. It has been observed that slight changes in the chemical structure of polyamines can induce large changes in their biological efficacy, and that spermine-type compounds with a 3-3-3 or a 3-4-3 carbon skeleton are particularly effective anti-tumor agents. ${ }^{29}$ The PEG-conjugate 9 was prepared for comparison purposes, since a PEG group normally increases the solubility and cellular uptake of porphyrin macrocycles, ${ }^{52}$ and it is often used as a spacer in cell-targeted porphyrin-peptide and - antibody conjugates.

All porphyrin conjugates were structurally characterized by NMR, MS, and UV-Vis spectroscopy. The partition coefficient $(\log P)$ values between 1-octanol and HEPES buffer (pH 7.4) were obtained using the shaking-flak method ${ }^{46}$ and are shown in Table 1. The hydrophobic character for the conjugates follows the order $\mathbf{7}<\mathbf{4} \sim 3<\mathbf{6}<\mathbf{5}<8<\mathbf{2} \sim 9$, depending on the number of amine groups and the carbon skeleton of the polyamine. The most hydrophobic polyamine conjugate was found to be $\mathbf{2}$ with a $\log P$ value similar to that of the PEG conjugate $\mathbf{9}$, whereas $\mathbf{7}$ bearing a spermine group was the least hydrophobic of this series.

\section{Cell culture}

Cytotoxicity-The concentration-dependent dark and phototoxicity of all conjugates $2-9$ were investigated in T98G cells and the results are shown in Figures 1 and 2, respectively. All conjugates showed low cytotoxicity in the dark, with determined $\mathrm{IC}_{50}(50 \%$ inhibition of cell proliferation based on dose-response curves) $>250 \mu \mathrm{M}$; of all conjugates, the PEGporphyrin 9 showed the highest dark toxicity with determined $\mathrm{IC}_{50}=296 \mu \mathrm{M}$. Upon exposure to $1.5 \mathrm{~J} / \mathrm{cm}^{2}$, the spermine derivatives $7,8,6$ and 5 were found to be the most toxic with $\mathrm{IC}_{50}=40,41,64$ and $87 \mu \mathrm{M}$, respectively. Compound toxicity is a key limiting factor that can prevent potential new boronated drugs from becoming practically useful in a clinical setting because of the high boron concentration requirement in BNCT. Significant toxicities ( $\mathrm{IC}_{50}<25 \mu \mathrm{M}$ in $\mathrm{F} 98$ rat glioma cells) were reported for $o$-carborane-containing derivatives of spermidine and spermine, and in particular for the terminally (rather than internally) $\mathrm{N}$-substituted derivatives. ${ }^{35}$ In contrast all our conjugates showed very low cytotoxicity in human glioma T98G cells, maybe as a result from attachment of the carborane clusters to the porphyrin macrocycle rather than directly to the polyamine chain, and the use of closo-1,12- (para) rather than closo-1,2- (ortho) dicarbadodecaboranes; in the $o$-carborane clusters the boron atoms bound to both carbons are highly susceptible to nucleophilic attack by amine groups, producing the corresponding negatively charged nido-1,2-dicarbaundecaboranes, therefore changing the overall charge and lipophilicity of the conjugates. ${ }^{51}$

Time-dependent uptake-The time-dependent uptake of porphyrin conjugates was evaluated at a concentration of $10 \mu \mathrm{M}$ over a time period of $24 \mathrm{~h}$ and the results obtained are shown in Figure 3. The extent of cellular uptake followed the order $7>6>3 \sim 4>5>8>2$ $\sim 9$, generally increasing with the hydrophilicity of the conjugates. All polyamine conjugates with the exception of $\mathbf{2}$ showed better plasma membrane permeability and uptake into T98G cells compared with the PEG conjugate $\mathbf{9}$; the compound taken up the most by cells at all 
time points investigated was spermine conjugate $\mathbf{7}$, about 12 times more than $\mathbf{9}$. This result might be due to active transport via the polyamine transporter system, particularly for conjugates 7 and $\mathbf{6}$ containing a 3-4-3 and 3-3-3 carbon backbone, and for 3 and $\mathbf{4}$ due to their larger number of nitrogens; such derivatives have been found in structure-activity studies to accumulate in cells via the polyamine transport system, ${ }^{29,53}$ although recent studies found no effects in the cellular uptake of polyamine-substituted phthalocyanines upon addition of spermidine or a-difluoromethylornithine. ${ }^{54}$ Nevertheless, the protonation of polyamines under physiological conditions (the pKa of the amine groups are in the range $7-10)^{55}$ likely favors interactions with membrane-containing phosphate groups inducing higher cellular uptake compared with the PEG conjugate. The plasma membrane of tumor cells usually contains higher net negative charge compared with normal cells, due to the over-expression of polysialic acid residues. ${ }^{56}$ In the polyamine-porphyrin conjugates the cationic polyamine groups likely facilitate binding to the negatively charged tumor cell plasma membranes, while the hydrophobic nature of the fluorinated porphyrin and carborane moieties additionally favor penetration of the conjugate through the lipid membrane. Our results suggest that polyamines, in particular derivatives of spermine, can serve as carriers of boronated porphyrins for BNCT, enhancing boron transport across plasma membranes and delivery into tumor cells. Furthermore, a polyamine group might be more effective than a PEG as a linker, in the synthesis of porphyrin-peptide conjugates. ${ }^{16}$

Subcellular Localization-Fluorescence microscopy was used to examine the intracellular localization of all conjugates in live cells. Human HEp2 rather than T98G cells were used in these studies because they adhere and spread nicely on glass cover slips, thus facilitating the imaging process. The organelle specific fluorescent probes ERTracker Blue/ White (ER), MitoTracker Green (mitochondria), BODIPY-FL Ceramide (Golgi), and LysoSensor Green (lysosomes) were used in overlay experiments, as shown in Figures 4-10. Figure S9 of the Supporting Information shows the corresponding images for the PEGconjugate 9. All conjugates were found to preferentially localize in the cell ER, as seen by the purple color in Figures 4d-10d. In addition, the polyamine conjugate 2 and the PEG conjugate 9 were also found to localize in mitochondria. We have previously observed that a porphyrin containing one PEG group localized subcellularly in the ER and mitochondria. ${ }^{52}$ Minor sites of localization for the polyamine conjugates were the Golgi and the cell lysosomes. Both negatively and positively charged carboranylporphyrins have been observed to localize mainly in the cell lysosomes, probably as a result of an endocytic mechanism of uptake. ${ }^{10,57}$ However, we believe that the presence of the polyamine and PEG groups favors localization of conjugates 2-9 in the ER, an important target in PDT ${ }^{58}$ and probably also in BNCT. Although the cell nuclei were apparently not targeted by the polyamine conjugates, the delivery of boron to their vicinity, localized in the ER, Golgi and lysosomes, might enhance the biological efficacy of these agents.

\section{Conclusions}

A series of fluorinated porphyrin-polyamine conjugates containing para-carborane clusters were synthesized in high yields via nucleophilic substitution of the $p$-phenyl fluorides of TPPF, and investigated as boron carriers for BNCT. para-Carborane clusters were used rather than the most common ortho-carboranes, due to their higher stability in the presence of nucleophilic amine groups. The hydrophobic character of the conjugates was investigated by determining the logarithm of their partition coefficient $(\log P)$ between 1-octanol and buffered water $(\mathrm{pH}=7.4)$. The most hydrophilic spermine-porphyrin conjugate 7 ( $\log P=$ 1.06) accumulated the most within human glioma T98G cells of all conjugates studied, about 12 -fold more than a pegylated-porphyrin derivative. This might be due to the target of the polyamine transport system, in spite of the bulky carboranylporphyrin, and/or to favorable interactions between the cationic polyamine chain and the negatively charged 
plasma membranes, and the overall lipophilic character of the conjugates. All polyamine conjugates showed very low dark toxicities $\left(\mathrm{IC}_{50}>400 \mu \mathrm{M}\right.$, lower than the PEG conjugate with determined $\mathrm{IC}_{50}=296 \mu \mathrm{M}$ ), a critical feature for potential boron delivery agents because of the very high boron concentration requirement in BNCT $(20-35 \mu \mathrm{g} / \mathrm{g})$. In addition all polyamine-porphyrin conjugates showed relatively low phototoxicity, the most phototoxic were the spermine derivatives $\mathbf{5 , 6}, \mathbf{7}$ and $\mathbf{8}$ with determined $\mathrm{IC}_{50}=87,64,40$ and $41 \mu \mathrm{M}$, respectively, at $1.5 \mathrm{~J} / \mathrm{cm}^{2}$, further indicating their usefulness as boron carriers for BNCT. In contrast to most currently known carboranylporphyrins, the main intracellular sites of localization for all conjugates were the ER; in addition, the polyamine conjugates were also observed in the Golgi and lysosomes. Among the polyamines, the spermine derivatives containing a 3-4-3 or 3-3-3 carbon skeleton are the most efficient at increasing cellular uptake and therefore are the most promising as boron delivery agent for BNCT.

\section{Supplementary Material}

Refer to Web version on PubMed Central for supplementary material.

\section{Acknowledgments}

This work was supported by the National Institutes of Health, grant number R21 CA139385.

\section{References}

1. Sivaev IB, Bregadze VI. Polyhedral boranes for medical applications: current status and perspectives. Eur J Inorg Chem. 2009; 11:1433-1450.

2. Vicente, MGH.; Sibrian-Vazquez, M. Syntheses of boronated porphyrins and their application in BNCT. In: Kadish, KM.; Smith, KM.; Guilard, R., editors. The Handbook of Porphyrin Science. Vol. 4. World Scientific Publishers; Singapore: 2010. p. 191-248.

3. Hawthorne MF. The role of chemistry in the development of boron neutron-capture therapy of cancer. Angew Chem Int Ed Eng. 1993; 32:950-984.

4. Soloway AH, Tjarks W, Barnum BA, Rong FG, Barth RF, Codogni IM, Wilson JG. The chemistry of neutron capture therapy. Chem Rev. 1998; 98:1515-1562. [PubMed: 11848941]

5. Barth RF, Coderre JA, Vicente MGH, Blue TE. Boron neutron capture therapy of cancer: Current status and future prospects. Clin Cancer Res. 2005; 11:3987-4002. [PubMed: 15930333]

6. Hopewell JW, Gorlia T, Pellettieri L, Giusti V, Stenstam BH, Skold K. Boron neutron capture therapy for newly diagnosed glioblastoma multiforme: an assessment of clinical potential. Appl Radiat Isot. 2011; 69:1737-1740. [PubMed: 21482122]

7. Kawabata S, Miyatake S, Hiramatsu R, Hirota Y, Miyata S, Takekita Y, Kuroiwa T, Kirihata M, Sakurai Y, Maruhashi A, Ono K. Phase II clinical study of boron neutron capture therapy combined with X-ray radiotherapy/temozolomide in patients with newly diagnosed glioblastoma multiformestudy design and current status report. Appl Radiat Isot. 2011; 69:1796-1799. [PubMed: 21459588]

8. Kankaanranta L, Seppälä T, Koivunoro H, Valimaki P, Beule A, Collan J, Kortesniemi M, UusiSimola J, Kotiluoto P, Auterinen I. L-boronophenylalanine-mediated boron neutron capture therapy for malignant glioma progressing after external beam radiation therapy: a Phase I study. Int J Radiat Oncol Biol Phys. 2011; 80:369-376. [PubMed: 21236605]

9. Yamamoto T, Nakai K, Nariai T, Kumada H, Okumura T, Mizumoto M, Tsuboi K, Zaboronok A, Ishikawa E, Aiyama H. The status of Tsukuba BNCT trial: BPA-based boron neutron capture therapy combined with X-ray irradiation. Appl Radiat Isot. 2011; 69:1817-1818. [PubMed: 21393005]

10. Hao E, Friso E, Miotto G, Jori G, Soncin M, Fabris C, Sibrian-Vazquez M, Vicente MGH. Synthesis and biological investigations of tetrakis ( $p$-carboranylthio-tetrafluorophenyl)chlorin (TPFC). Org Biomol Chem. 2008; 6:3732-3740. [PubMed: 18843403]

11. Dougherty TJ, Gomer CJ, Henderson BW, Jori G, Kessel D, Korbelik M, Moan J, Peng Q. Photodynamic therapy. J Natl Cancer Inst. 1998; 90:889-905. [PubMed: 9637138] 
12. Pandey RK. Recent advances in photodynamic therapy. J Porphyrins Phthalocyanines. 2000; 4:368-373.

13. Brown SB, Brown EA, Walker I. The present and future role of photodynamic therapy in cancer treatment. Lancet Oncology. 2004; 5:497-508. [PubMed: 15288239]

14. Huang Z. A review of progress in clinical photodynamic therapy. Tech Cancer Res Treat. 2005; 4:283-293.

15. Dozzo P, Koo MS, Berger S, Forte TM, Kahl SB. Synthesis, characterization, and plasma lipoprotein association of a nucleus-targeted boronated porphyrin. J Med Chem. 2005; 48:357359. [PubMed: 15658849]

16. Sibrian-Vazquez M, Hao E, Jensen TJ, Vicente MGH. Enhanced cellular uptake with a cobaltacarborane-porphyrin-HIV-1 Tat 48-60 conjugate. Bioconjugate Chem. 2006; 17:928-934.

17. Crossley EL, Ziolkowski EJ, Coderre JA, Rendina LM. Boronated DNA-binding compounds as potential agents for boron neutron capture therapy. Mini-Rev Med Chem. 2007; 7:303-313. [PubMed: 17346220]

18. Hartman T, Lundqvist H, Westlin JE, Carlsson J. Radiation doses to the cell nucleus in single cells and cells in micrometastases in targeted therapy with I-131 labeled ligands or antibodies. Int J Rad Oncol Biol Phys. 2000; 46:1025-1036.

19. Gryshuk A, Chen Y, Goswami LN, Pandey S, Missert JR, Ohulchanskyy T, Potter W, Prasad PN, Oseroff A, Pandey RK. Structure-activity relationship among purpurinimides and bacteriopurpurinimides: trifluoromethyl substituent enhanced the photosensitizing efficacy. J Med Chem. 2007; 50:1754-1767. [PubMed: 17371002]

20. Ko YJ, Yun KJ, Kang MS, Park J, Lee KT, Park SB, Shin JH. Synthesis and in vitro photodynamic activities of water-soluble fluorinated tetrapyridylporphyrins as tumor photosensitizers. Biorg Med Chem Lett. 2007; 17:2789-2794.

21. Miyashita M, Miyatake S, Imahori Y, Yokoyama K, Kawabata S, Kajimoto Y, Shibata MA, Otsuki Y, Kirihata M, Ono K, Kuroiwa T. Evaluation of fluoride-labeled boronophenylalanine-PET imaging for the study of radiation effects in patients with glioblastomas. J Neurooncol. 2008; 89:239-246. [PubMed: 18566749]

22. Cullis PM, Green RE, Merson-Davies L, Travis N. Probing the mechanism of transport and compartmentalisation of polyamines in mammalian cells. Chem Biol. 1999; 6:717-729. [PubMed: 10508681]

23. Carlisle DL, Devereux WL, Hacker A, Woster PM, Casero RA. Growth status significantly affects the response of human lung cancer cells to antitumor polyamine-analogue exposure. Clin Cancer Res. 2002; 8:2684-2689. [PubMed: 12171901]

24. Wang C, Delcros JG, Biggerstaff J, Phanstiel O IV. Molecular requirements for targeting the polyamine transport system. Synthesis and biological evaluation of polyamine-anthracene conjugates. J Med Chem. 2003; 46:2672-2682. [PubMed: 12801231]

25. Sol V, Lamarche F, Enache M, Garcia G, Granet R, Guilloton M, Blais JC, Krausz P. Polyamine conjugates of meso-tritolylporphyrin and protoporphyrin IX: Potential agents for photodynamic therapy of cancers. Bioorg Med Chem. 2006; 14:1364-1377. [PubMed: 16263292]

26. Hahn F, Schmitz K, Balaban TS, Brase S, Schepers U. Conjugation of spermine facilitates cellular uptake and enhances antitumor and antibiotic properties of highly lipophilic porphyrins. Chem Med Chem. 2008; 3:1185-1188. [PubMed: 18618563]

27. Garcia G, Sarrazy V, Sol V, Morvan CL, Granet R, Alves S, Krausz P. DNA photocleavage by porphyrin-polyamine conjugates. Bioorg Med Chem. 2009; 17:767-776. [PubMed: 19097912]

28. Sarrazy V, Garcia G, Bakidi JPM, Morvan CL, Begaud-Grimaud G, Granet R, Sol V, Krausz P. Photodynamic effects of porphyrin-polyamine conjugates in human breast cancer and keratinocyte cell lines. J Photochem Photobiol B: Biol. 2011; 103:201-206.

29. Casero RA Jr, Woster PM. Recent advances in the development of polyamine analogues as antitumor agents. J Med Chem. 2009; 52:4551-4573. [PubMed: 19534534]

30. Papadopoulou MV, Rosenzweig HS, Bloomer WD. Synthesis of a novel nitroimidazolespermidine derivative as a tumor-targeted hypoxia-selective cytotoxin. Bioorg Med Chem Lett. 2004; 14:1519-1522. [PubMed: 15006394] 
31. Chadwick J, Jones M, Mercer AE, Stocks PA, Ward SA, Park BK, O’Neill PM. Design, synthesis and antimalarial/anticancer evaluation of spermidine linked artemisinin conjugates designed to exploit polyamine transporters in Plasmodium falciparum and HL-60 cancer cell lines. Bioorg Med Chem. 2010; 18:2586-2597. [PubMed: 20227283]

32. Vida N, Svobodová H, Rárová L, Drašar P, Šaman D, Cvačka J, Wimmer Z. Polyamine conjugates of stigmasterol. Steroids. 2012; 77:1212-1218. [PubMed: 22850319]

33. Zhu Q, Jin L, Casero RB. Role of ornithine decarboxylase in regulation of estrogen receptor alpha expression and growth in human brest cancer cells. Brest Cancer Res Treat. 2012; 136:57-66.

34. Travares AR, Kanashiro S, Castilho CC, Vianello F, Lima GPP. Effect of spermidine on ornamental bromeliad cultured in vitro. Afr J Biotechnol. 2012; 11:13988-13991.

35. Cai J, Soloway AH, Barth RF, Adams DM, Hariharan JR, Wyzlic IM, Radcliffe K. Boroncontaining polyamines as DNA targeting agents for neutron capture therapy of brain tumors: Synthesis and biological evaluation. J Med Chem. 1997; 40:3887-3896. [PubMed: 9397169]

36. Martin B, Posseme F, Le Barbier C, Carreaux F, Carboni B, Seiler N, Moulinoux JP, Delcros JG. $\mathrm{N}$-Benzylpolyamines as vectors of boron and fluorine for cancer therapy and imaging: synthesis and biological evaluation. J Med Chem. 2001; 44:3653-3664. [PubMed: 11606130]

37. Ghaneolhosseini H, Tjarks W, Sjoberg S. Synthesis of novel boronated acridines- and spermidines as possible agents for BNCT. Tetrahedron. 1998; 54:3877-3884.

38. Zhuo JC, Cai J, Soloway AH, Barth RF, Adams DM, Ji W, Tjarks W. Synthesis and biological evaluation of boron-containing polyamines as potential agents for neutron capture therapy of brain tumors. J Med Chem. 1999; 42:1282-1292. [PubMed: 10197971]

39. El-Zaria ME, Doerfler U, Gabel D. Synthesis of (aminoalkylamine)- $N$ aminoalkyl)azanonaborane(11) derivatives for boron neutron capture therapy. J Med Chem. 2002; 45:5817-5819. [PubMed: 12477367]

40. Yang XB, Wang Q, Huang Y, Fu PH, Zhang JS, Zeng RQ. Synthesis, DNA interaction and antimicrobial activities of copper (II) complexes with Schiff base ligands derived from kaempferol and polyamines. Inorg Chem Commun. 2012; 25:55-59.

41. Iacomino G, Picariello G, D'Agostino L. DNA and nuclear aggregates of polyamines. Biochim Biophys Acta - Mol Cell Res. 2012; 1823:1745-1755.

42. Tüxen J, Eibenberger S, Gerlich S, Arndt M, Mayor M. Highly fluorous porphyrins as model compounds for molecule interferometry. Eur J Org Chem. 2011; 25:4823-4833.

43. Samaroo D, Soll CE, Todaro LJ, Drain CM. Efficient microwave-assisted synthesis of aminesubstituted tetrakis(pentafluorophenyl)porphyrin. Organic Lett. 2006; 8:4985-4988.

44. Geall AJ, Taylor RJ, Earll ME, Eaton MAW, Blagbrough IS. Synthesis of cholesteryl polyamine carbamates: pKa studies and condensation of calf thymus DNA. Bioconjugate Chem. 2000; 11:314-326.

45. Ujváry I, Nachman RJ. Synthesis of heterobifunctional p-carborane derivatives. 3-[12(Mercaptomethyl)-1,12-dicarba-closo-dodecaboran(12)-1-yl]propionic acid. Tetrahedron Lett. 1999; 40:5147-5149.

46. Kepczynski M, Pandian RP, Smith KM, Ehrenberg B. Do liposome-binding constants of porphyrins correlate with their measured and predicted partitioning between octanol and water? Photochem Photobiol. 2002; 76:127-134. [PubMed: 12194207]

47. Volz H, Schneckenburger S. meso-Substituted Porphyrins. 6. An Efficient Synthesis of 5,10,15,20Tetrakis-(pentafluorphenyl)-porphyrin. J Prak Chemie/Chemiker-Zeitung. 1993; 335:283-284.

48. Shaw SJ, Elgie KJ, Edwards C, Boyle RW. Mono-(pentafluorophenyl)porphyrins - useful intermediates in the regioselective synthesis of multifunctionalised porphyrins. Tetrahedron Lett. 1999; 40:1595-1596.

49. Suzuki M, Shimizu S, Shin JY, Osuka A. Regioselective nucleophilic substitution reaction of meso-hexakis(pentafluorophenyl) substituted [26]hexaphyrin. Tetrahedron Lett. 2003; 44:45974601.

50. Samaroo D, Vinodu M, Chen X, Drain CM. meso-Tetra(pentafluorophenyl)porphyrin as an efficient platform for combinatorial synthesis and the selection of new photodynamic therapeutics using a cancer cell line. J Comb Chem. 2007; 9:998-1011. [PubMed: 17877415] 
51. Bregadze VI. Dicarba-closo-dodecaboranes $\mathrm{C}_{2} \mathrm{~B}_{10} \mathrm{H}_{12}$ and their derivatives. Chem Rev. 1992; 92:209-223.

52. Sibrian-Vazquez M, Jensen TJ, Vicente MGH. Synthesis and cellular studies of PEGfunctionalized meso-tetraphenylporphyrins. J Photochem Photobiol B: Biol. 2007; 86:9-21.

53. Delcros JG, Tomasi S, Duhieu S, Foucault M, Martin B, Le Roch M, Eifler-Lima V, Renault J, Uriac P. Effect of polyamine homologation on the transport and biological properties of heterocyclic amidines. J Med Chem. 2006; 49:232-245. [PubMed: 16392808]

54. Jiang XJ, Yeung SL, Lo PC, Fong WP, Ng DKP. Phthalocyanine-polyamine conjugates as highly efficient photosensitizers for photodynamic therapy. J Med Chem. 2011; 54:320-330. [PubMed: 21138268]

55. Agostinelli E, Marques MP, Calheiros R, Gil FP, Tempera G, Viceconte N, Battaglia V, Grancara S, Toninello A. Polyamines: fundamental characters in chemistry and biology. Amino Acids. 2010; 38:393-403. [PubMed: 20013011]

56. Janas T, Nowotarski K, Janas T. The effect of long-chain bases on polysialic acid-mediated membrane interactions. Biochim Biophys Acta. 2011; 1808:2322-2326. [PubMed: 21616054]

57. Easson MW, Fronczek FR, Jensen TJ, Vicente MGH. Synthesis and in vitro properties of trimethylamine- and phosphonate-substituted carboranylporphyrins for application in BNCT. Bioorg Med Chem. 2008; 16:3191-3208. [PubMed: 18178445]

58. Kessel D. Correlation between subcellular localization and photodynamic efficacy. J Porphyrins Phthalocyanines. 2004; 8:1009-1014. 


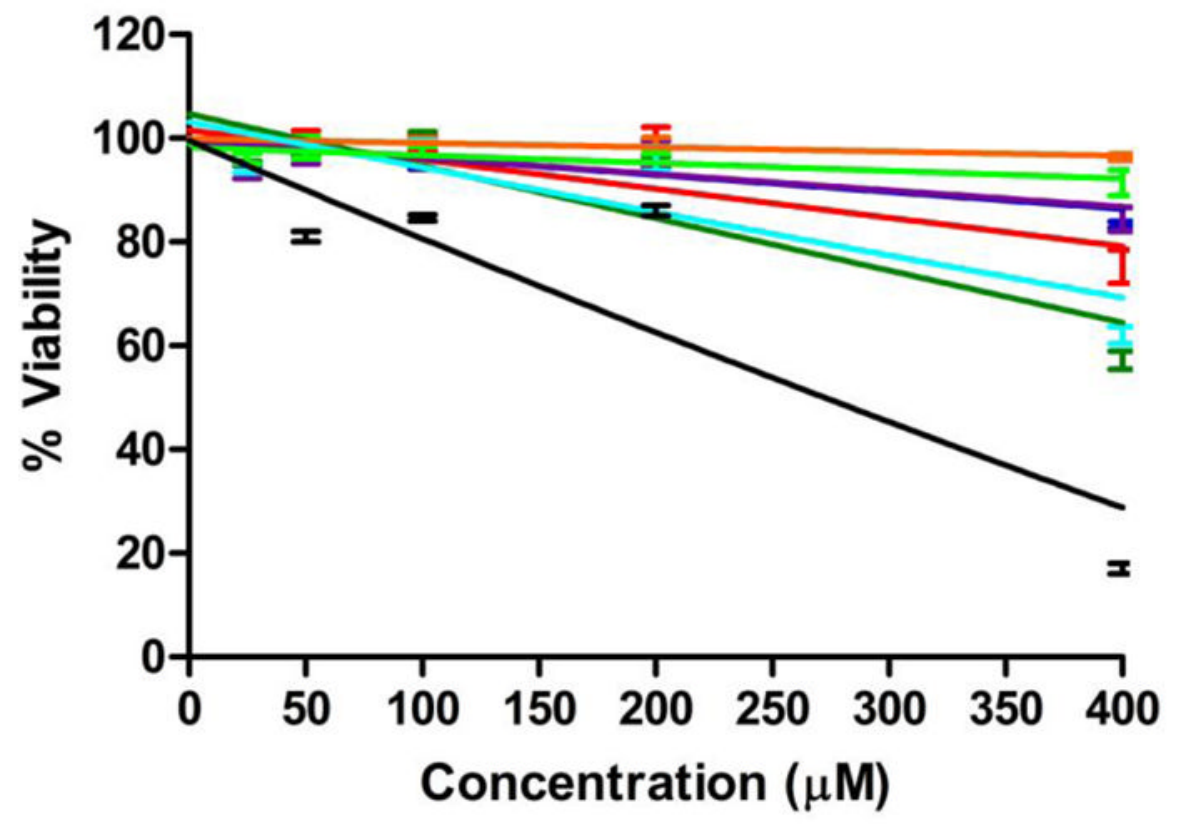

Figure 1.

Dark cytotoxicity of conjugates $\mathbf{2}$ (red), $\mathbf{3}$ (dark blue), $\mathbf{4}$ (purple), $\mathbf{5}$ (dark green), $\mathbf{6}$ (orange), 7 (light blue), 8 (light green) and $\mathbf{9}$ (black) toward human glioma T98G cells using a Cell Titer Blue assay. 


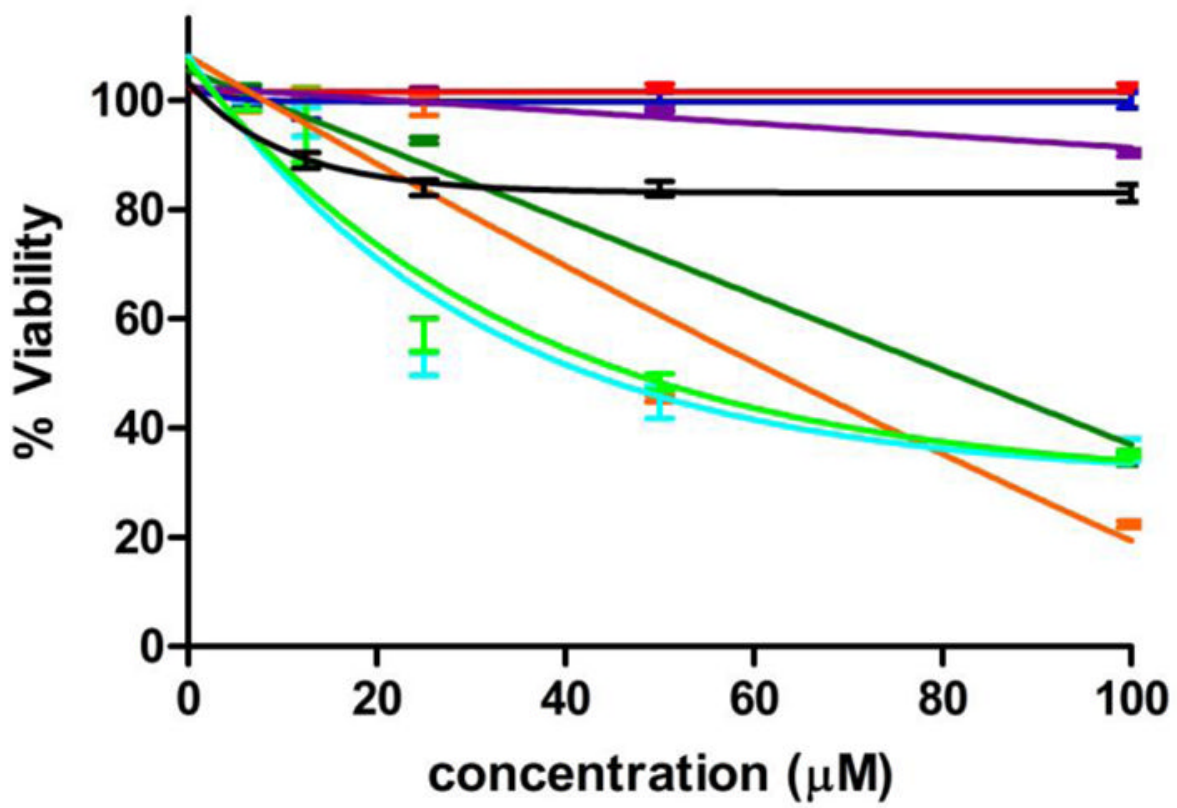

Figure 2.

Phototoxicity $\left(1.5 \mathrm{~J} / \mathrm{cm}^{2}\right.$ ) of conjugates $\mathbf{2}$ (red), $\mathbf{3}$ (dark blue), 4 (purple), $\mathbf{5}$ (dark green), $\mathbf{6}$ (orange), 7 (light blue), $\mathbf{8}$ (light green) and $\mathbf{9}$ (black) toward human glioma T98G cells using a Cell Titer Blue assay. 


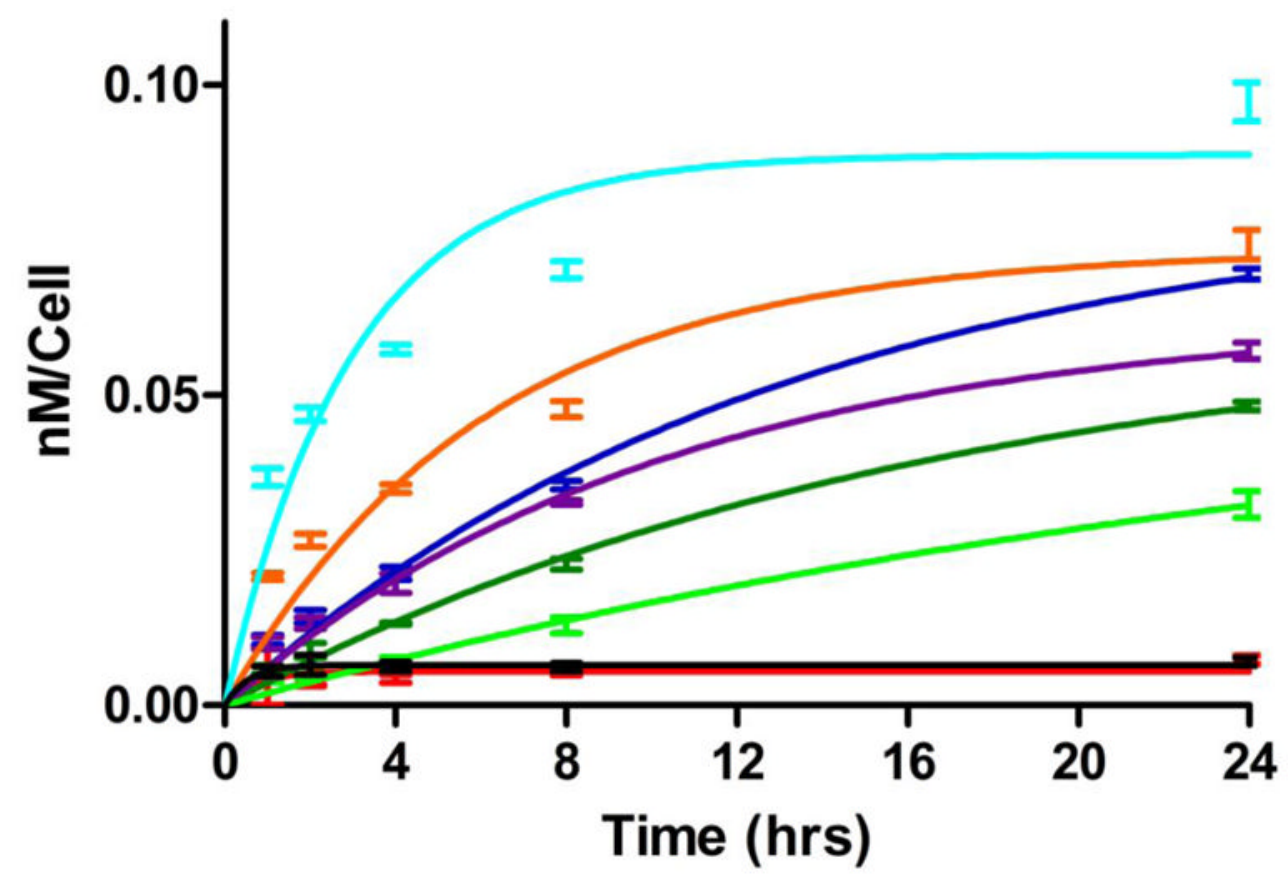

Figure 3.

Time-dependent uptake of conjugates 2 (red), $\mathbf{3}$ (dark blue), $\mathbf{4}$ (purple), $\mathbf{5}$ (dark green), $\mathbf{6}$ (orange), 7 (light blue), 8 (light green) and $\mathbf{9}$ (black) at $10 \mu \mathrm{M}$ by human glioma T98G cells. 


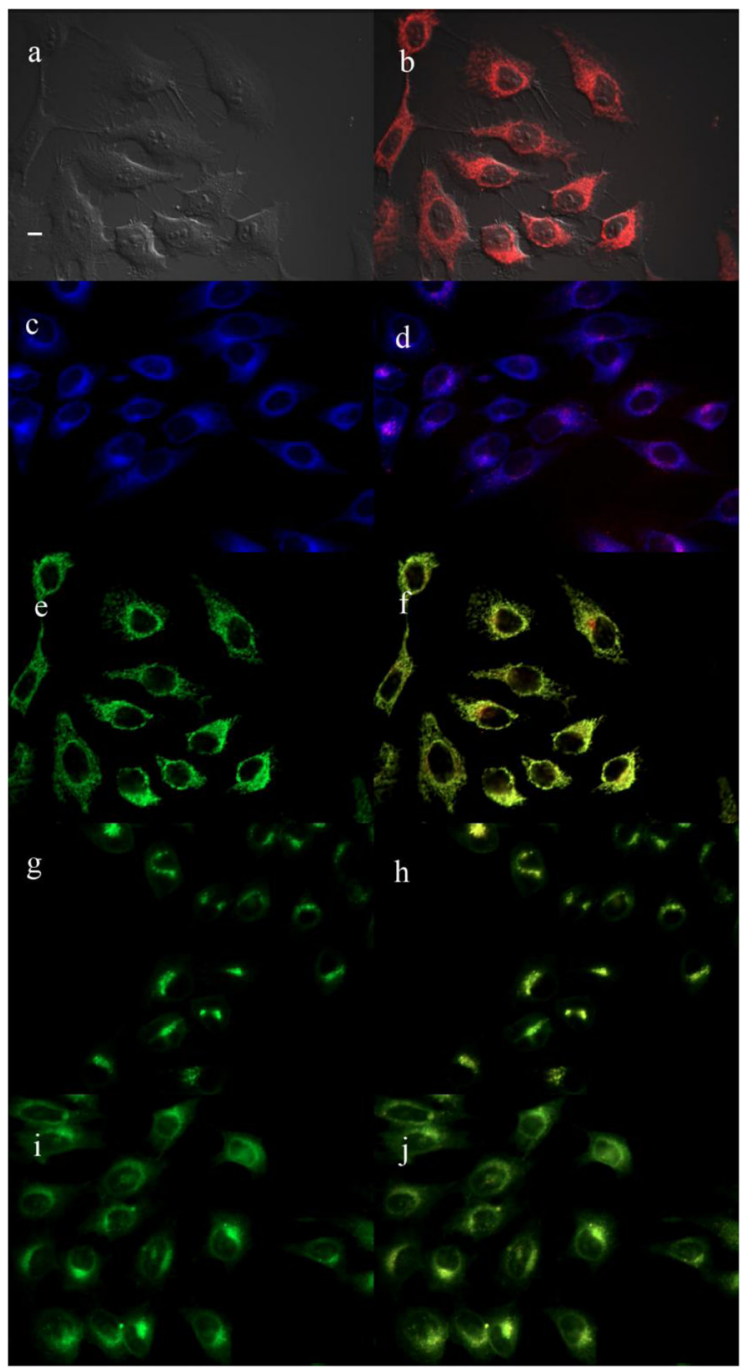

Figure 4.

Subcellular fluorescence of conjugate 2 in HEp2 cells at $10 \mu \mathrm{M}$ for $6 \mathrm{~h}$. (a) Phase contrast, (b) overlay of the $\mathbf{2}$ fluorescence and phase contrast, (c) ER Tracker Blue/White fluorescence, (e) MitoTracker Green fluorescence, (g) BODIPY Ceramide, (i) LysoSensor Green fluorescence, and (d, f, h, j) overlays of organelle tracers with 2 fluorescence. Scale bar: $10 \mu \mathrm{m}$. 


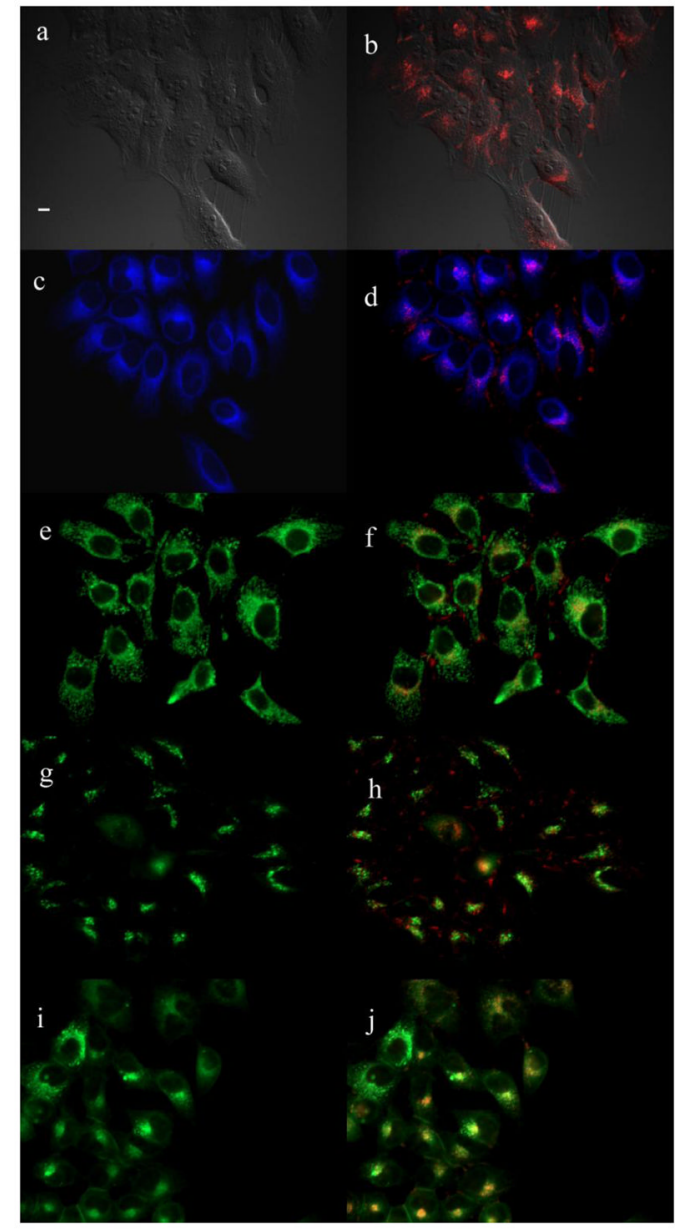

Figure 5.

Subcellular fluorescence of conjugate 3 in HEp2 cells at $10 \mu \mathrm{M}$ for $6 \mathrm{~h}$. (a) Phase contrast, (b) overlay of the $\mathbf{3}$ fluorescence and phase contrast, (c) ER Tracker Blue/White fluorescence, (e) MitoTracker Green fluorescence, (g) BODIPY Ceramide, (i) LysoSensor Green fluorescence, and (d, f, h, j) overlays of organelle tracers with $\mathbf{3}$ fluorescence. Scale bar: $10 \mu \mathrm{m}$. 


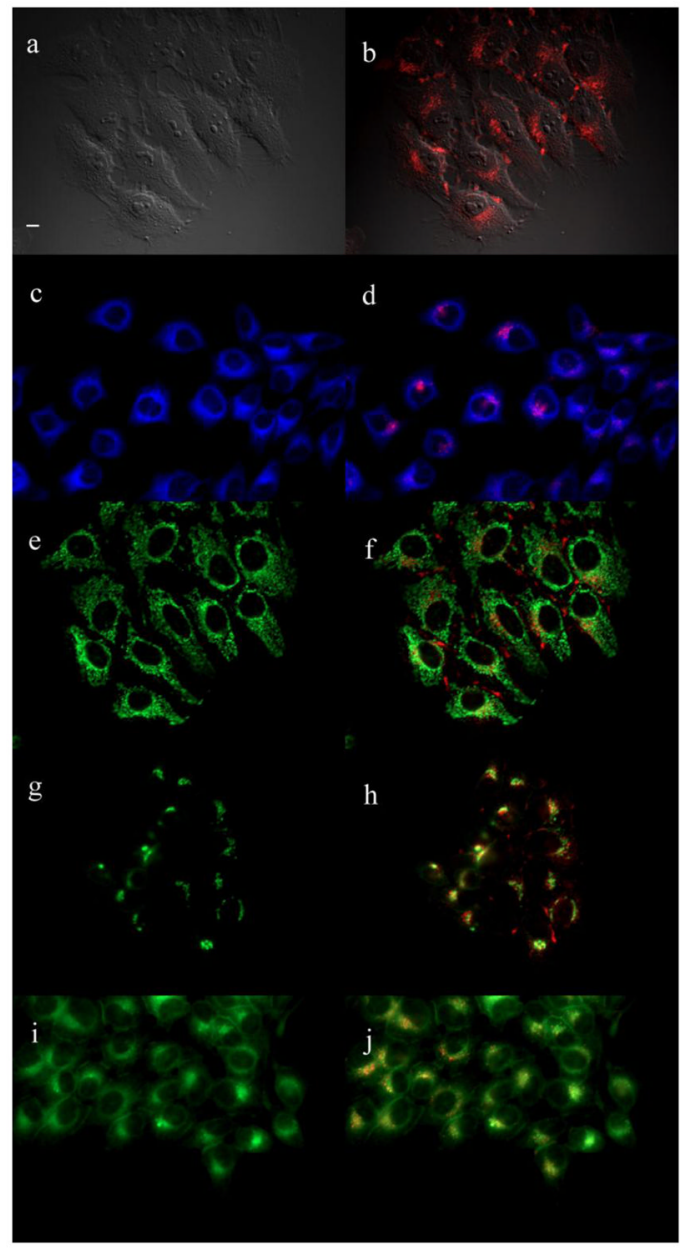

Figure 6.

Subcellular fluorescence of conjugate 4 in HEp2 cells at $10 \mu \mathrm{M}$ for $6 \mathrm{~h}$. (a) Phase contrast, (b) overlay of the $\mathbf{4}$ fluorescence and phase contrast, (c) ER Tracker Blue/White fluorescence, (e) MitoTracker Green fluorescence, (g) BODIPY Ceramide, (i) LysoSensor Green fluorescence, and (d, f, h, j) overlays of organelle tracers with $\mathbf{4}$ fluorescence. Scale bar: $10 \mu \mathrm{m}$. 


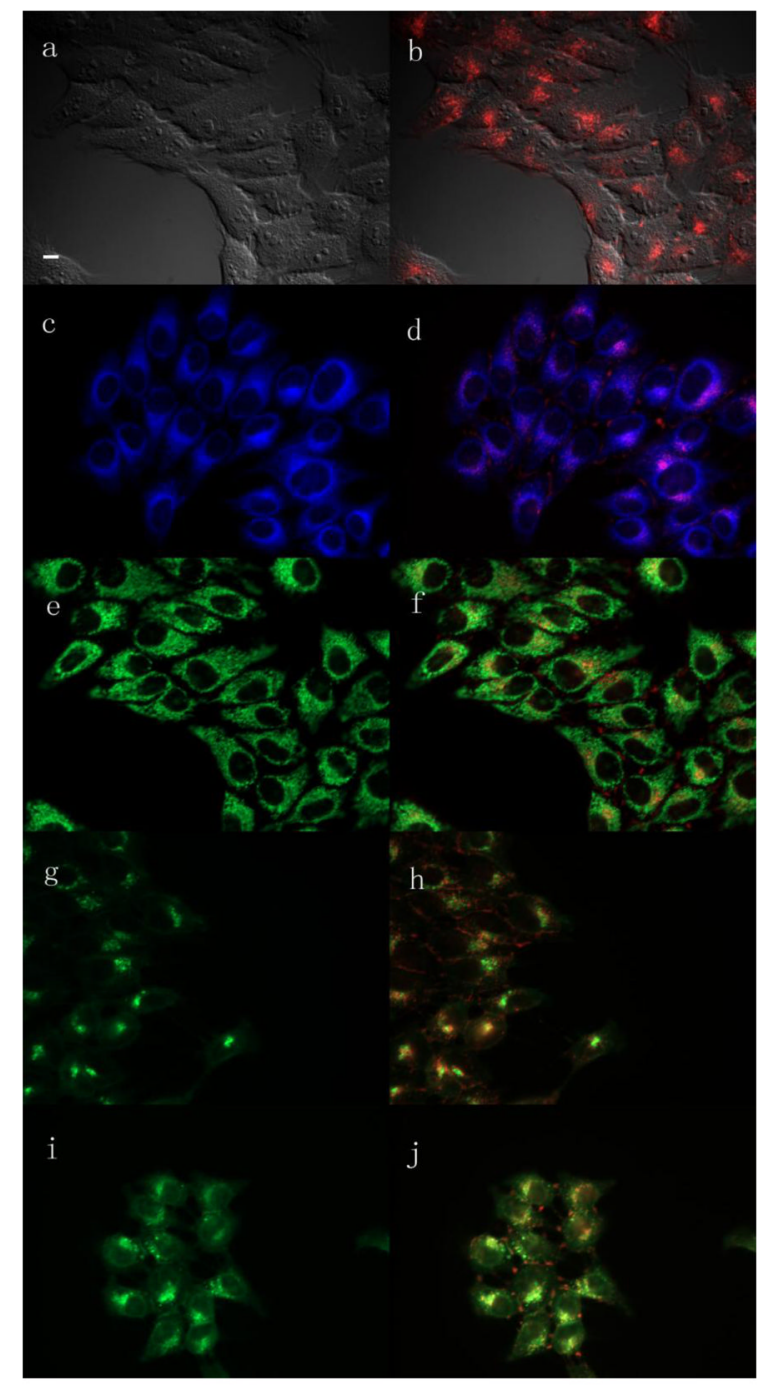

Figure 7.

Subcellular fluorescence of conjugate 5 in HEp2 cells at $10 \mu \mathrm{M}$ for $6 \mathrm{~h}$. (a) Phase contrast, (b) overlay of the $\mathbf{5}$ fluorescence and phase contrast, (c) ER Tracker Blue/White fluorescence, (e) MitoTracker Green fluorescence, (g) BODIPY Ceramide, (i) LysoSensor Green fluorescence, and (d, f, h, j) overlays of organelle tracers with 5 fluorescence. Scale bar: $10 \mu \mathrm{m}$. 


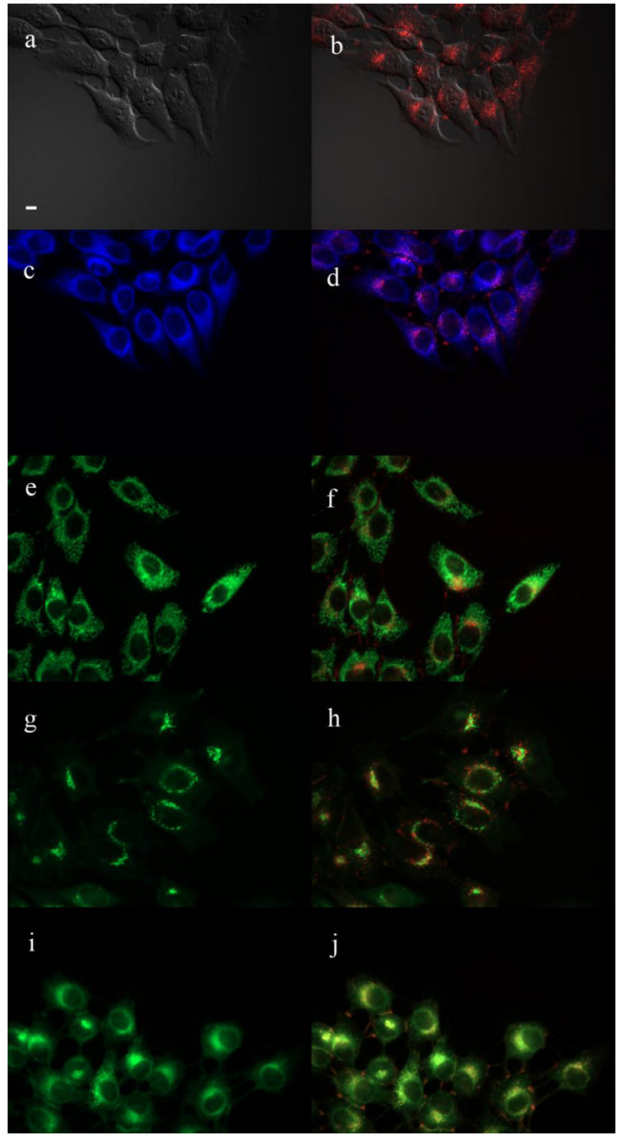

Figure 8.

Subcellular fluorescence of conjugate 6 in HEp2 cells at $10 \mu \mathrm{M}$ for $6 \mathrm{~h}$. (a) Phase contrast, (b) overlay of the 6 fluorescence and phase contrast, (c) ER Tracker Blue/White fluorescence, (e) MitoTracker Green fluorescence, (g) BODIPY Ceramide, (i) LysoSensor Green fluorescence, and (d, f, h, j) overlays of organelle tracers with 6 fluorescence. Scale bar: $10 \mu \mathrm{m}$. 


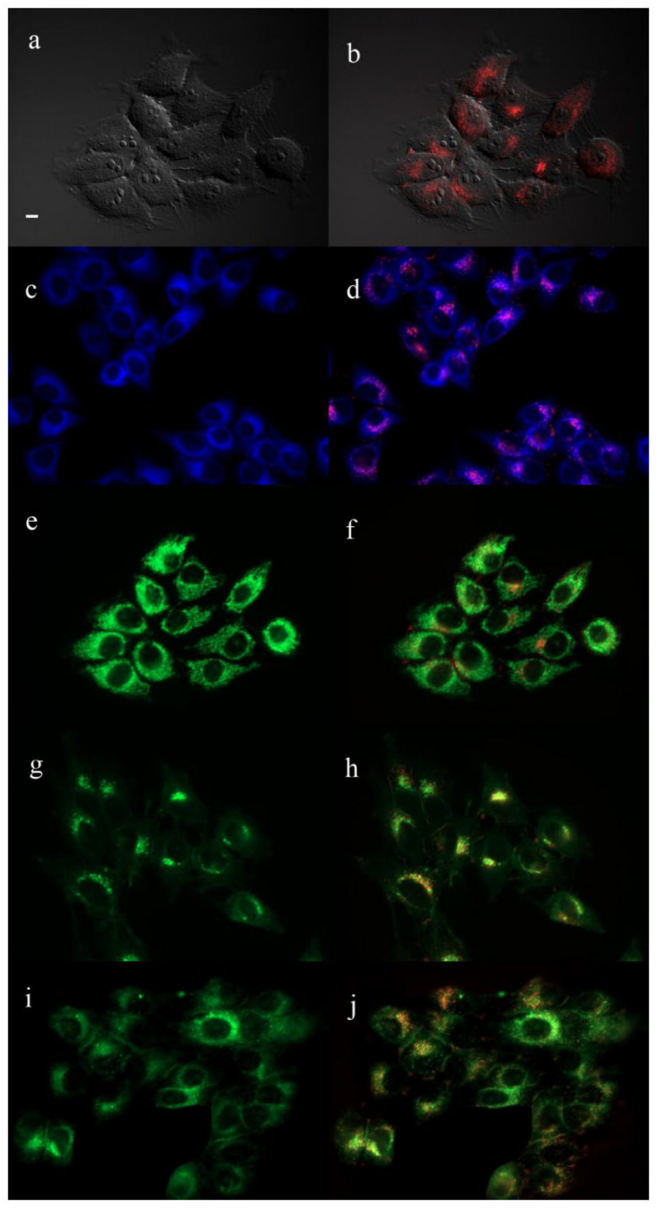

Figure 9.

Subcellular fluorescence of conjugate 7 in HEp2 cells at $10 \mu \mathrm{M}$ for $6 \mathrm{~h}$. (a) Phase contrast, (b) overlay of the 7 fluorescence and phase contrast, (c) ER Tracker Blue/White fluorescence, (e) MitoTracker Green fluorescence, (g) BODIPY Ceramide, (i) LysoSensor Green fluorescence, and (d, f, h, j) overlays of organelle tracers with 7 fluorescence. Scale bar: $10 \mu \mathrm{m}$. 


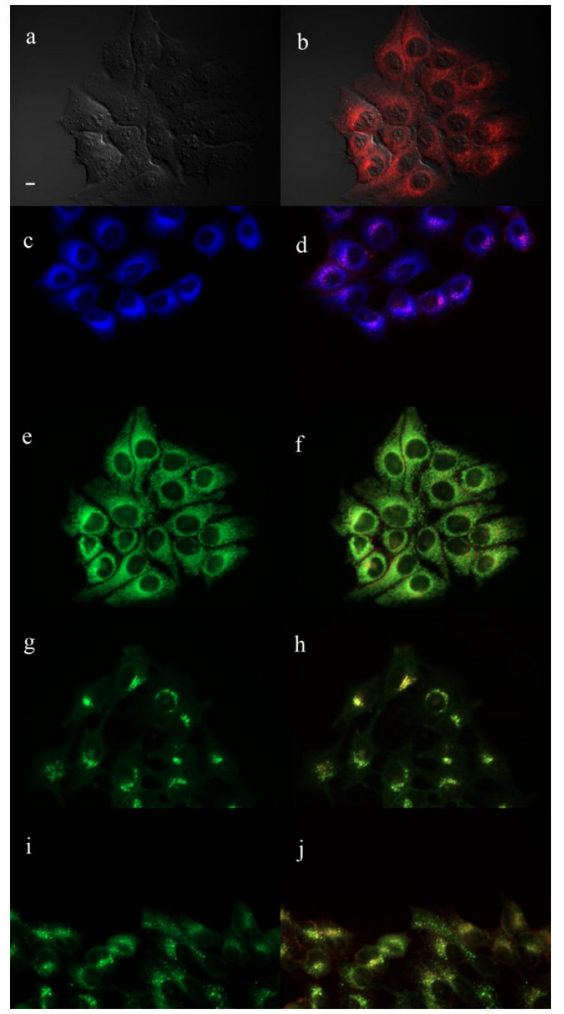

Figure 10.

Subcellular fluorescence of conjugate $\mathbf{8}$ in HEp2 cells at $10 \mu \mathrm{M}$ for $6 \mathrm{~h}$. (a) Phase contrast, (b) overlay of the $\mathbf{8}$ fluorescence and phase contrast, (c) ER Tracker Blue/White fluorescence, (e) MitoTracker Green fluorescence, (g) BODIPY Ceramide, (i) LysoSensor Green fluorescence, and (d, f, h, j) overlays of organelle tracers with $\mathbf{8}$ fluorescence. Scale bar: $10 \mu \mathrm{m}$. 


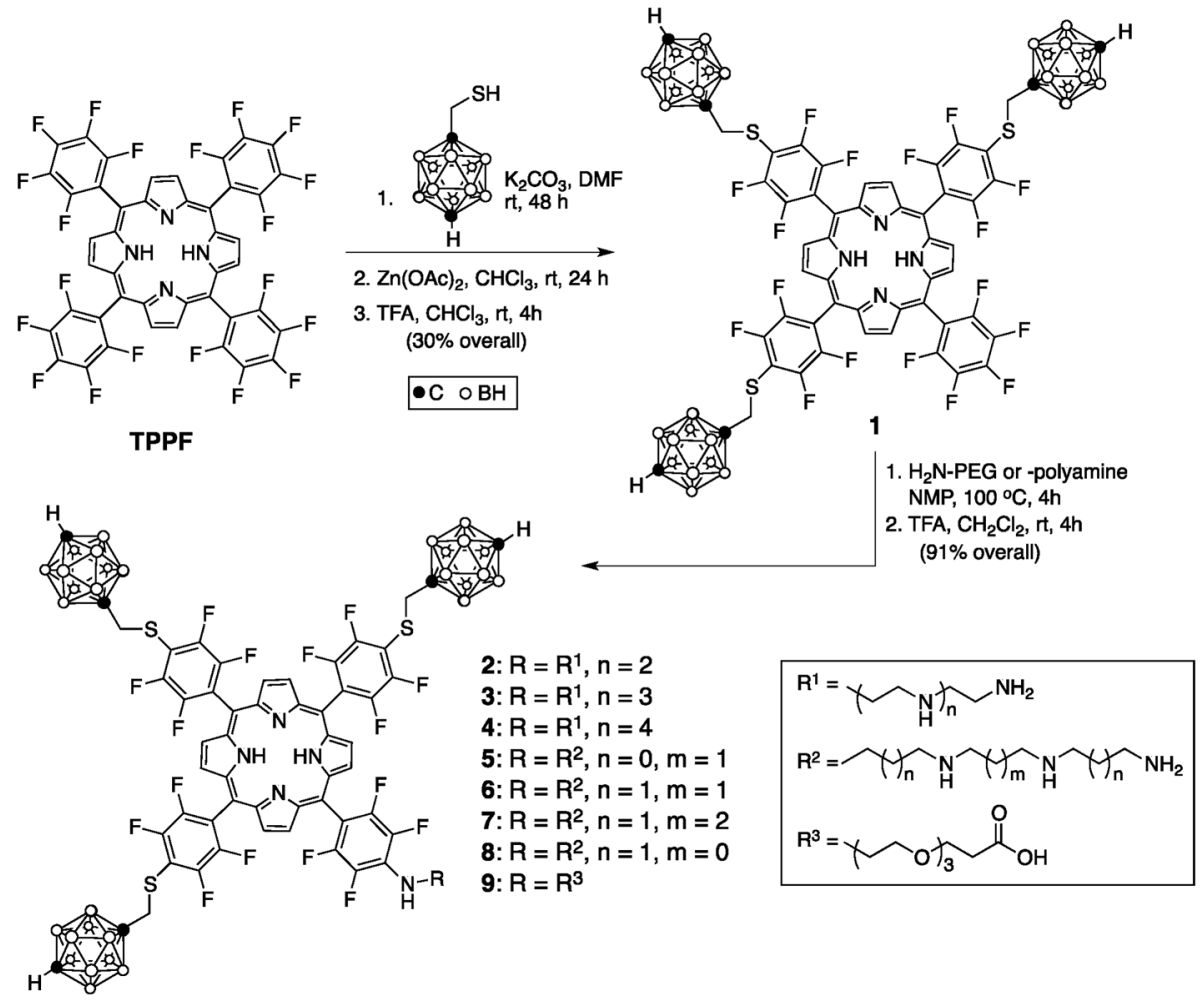

Scheme 1. 


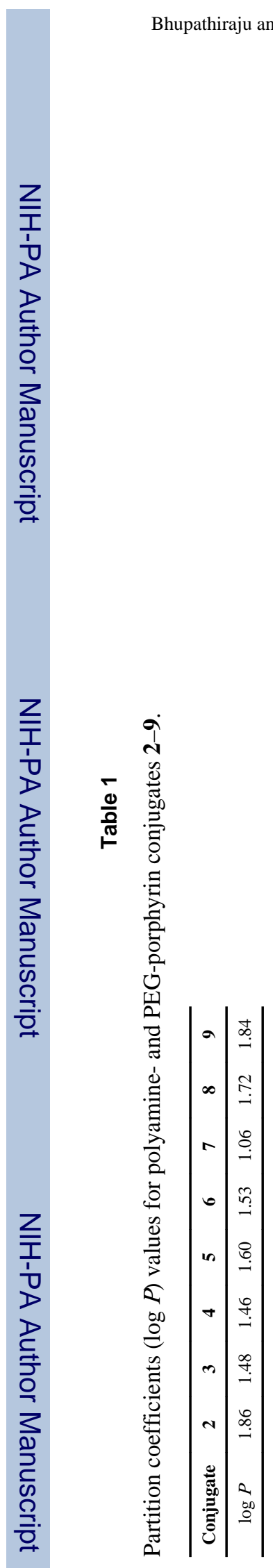

Bioorg Med Chem. Author manuscript; available in PMC 2014 January 15. 